\title{
Roughness effects on scalar transport
}

\author{
Zvi Hantsis and Ugo Piomelli周 \\ Department of Mechanical and Materials Engineering \\ Queen's University \\ 130 Stuart, McLaughlin Hall \\ Kingston, ON KYL 3N6 Canada
}

(Dated: October 29, 2020)

\begin{abstract}
We have studied the transport of a passive scalar for passive scalar with Prandtl numbers near unity in a plane channel with rough walls. The study was carried out by direct numerical simulations of the Navier-Stokes equations; an Immersed Boundary Method was used to model the roughness. The well-known departure from the Reynolds Analogy, which postulates similarity between the statistics of the scalar and the velocity, is verified. Townsend's similarity, suggesting the smooth-wall and rough-wall statistics collapse away from the wall is confirmed. The role of the form-induced production was a focus of this work. Additional form-induced contributions appear in the Reynolds-stress and scalar-variance budgets due to the roughness; they were quantified and compared between passive scalar and momentum. The form-induced production is more significant for the scalar variance than for the streamwise Reynolds stress, and could be the cause of the reduced damping of scalar fluctuations by the roughness. The sheltering caused by tall roughness elements decreases the mean gradients significantly but, in the case of the scalar, is countered by diffusion, so that the form-induced production is larger for the scalar variance. The implications of this finding are discussed.
\end{abstract}

\footnotetext{
*ugo@queensu.ca
} 


\section{INTRODUCTION}

Scalar transport occurs frequently in engineering applications and natural processes, from dispersion of particles and pollutants in the atmosphere to heat transfer in mechanical systems. It is, therefore, critical in many applications (particularly those involving heat transfer) to understand the phenomena that govern scalar transport.

One of the cornerstones of our knowledge of scalar transport is the Reynolds Analogy [1, 2], which relates the heat and momentum transport. This analogy is based on the observation that both momentum and heat transport depend on the same turbulent eddies and that the governing equations for momentum and scalar transport are nearly identical; if the Prandtl number (the ratio of viscosity to diffusivity) is equal to one, the only difference between them is the pressure gradient, which is absent in the scalar transport equation. Thus, it can be expected that the velocity and scalar profiles, properly normalized, are analogous, and that the ratio of heat transfer coefficient (the Stanton number) to the skin friction coefficient, known as the Reynolds-analogy ratio, is constant. The analogy was further extended, as additional similarities between momentum and scalar transport (mean stresses and fluxes, fluctuation intensities and Reynolds stresses and fluxes) were observed. The Reynolds analogy has been verified experimentally, and holds well in a variety of flows over hydraulically smooth surfaces [3-7].

Since all surfaces are hydraulically rough for sufficiently high Reynolds number, the understanding of roughness effects on scalar transport is important. While a very large body of work examines the momentum transport in the presence of roughness, fewer researchers have investigated the effect of roughness on scalar transport.

The effects of roughness on the momentum transport in boundary layers have been reviewed by Raupach et al. [8] and Jiménez [9]. The main ones are (1) an increase in the drag [10, 11]; (2) the amplification of the wall-normal and spanwise fluctuations, at the expense of the streamwise ones, resulting in decreased flow anisotropy [12 14]; (3) the breakup of the near-wall structures and modifications of the near-wall turbulence generation cycle [8, 15]. Townsend [16] proposed the hypothesis that the outer layer is insensitive to the inner region, except in the role it plays in setting the length and velocity scales for the outer flow. This implies that roughness effects are confined to a layer near the roughness, and that at some distance above the roughness crest the turbulent stresses, scaled by the friction velocity, 
collapse on the smooth data. Several studies confirmed this hypothesis, determining this distance to be roughly 3-5 times the equivalent sand-grain roughness height $\left(k_{s}\right)$ [16, 17]. Thus, roughness modifies the flow in the roughness sublayer and determines the velocity scale, but does not directly affect the stresses in the outer flow.

Early experimental studies of heat transfer in smooth- and rough-wall boundary layer quantified the relation between the Stanton number and the skin friction coefficient as a function of the Reynolds and Prandtl numbers [6, 18 21]. The studies concluded that roughness increases the Stanton number, but this increase is accompanied by a larger increase in the skin-friction coefficient. It was observed that, while the Reynolds Analogy holds well for hydraulically-smooth walls without significant pressure gradients, it is not valid for rough wall pipes, channels and boundary layers. Dipprey and Sabersky [19] conjectured that this breakdown is due to the increasing importance of the pressure mechanism in the momentum transfer (i.e., in the drag force) without a corresponding mechanism in the transport of scalar.

Direct Numerical Simulations (DNS) have also been used to investigate scalar transport. Several studies ([22 29] among others) have highlighted the physics of the near-wall region in smooth-wall flows and compared spectra and budgets for velocity and scalar. It was found that the scalar fluctuation and wall-normal fluxes were closely correlated with the streamwise velocity fluctuation and wall-normal stress, respectively, particularly in the nearwall region. The kinetic energy and scalar variance spectra were found to be reasonably analogous in both inner and outer regions and in both streamwise and spanwise directions. These studies also examined the scalar-fluctuation budgets, remarking on the similarity with that of the streamwise velocity fluctuation. Specifically, in channel flow the ratio between the characteristic time scales of the velocity and scalar fluctuations was found to be approximately constant over the greater part of the channel when $R e$ is sufficiently large and $\operatorname{Pr}$ is near unity. Differences between the scalar large-scale structures and their streamwise velocity counterparts were observed, as the scalar structures were both larger in the spanwise direction and steeper in inclination. Another key observation apparent in all the studies is the sharper interface of the scalar structures, emphasizing the unmixed nature of the scalar.

Nagano et al. [30], Leonardi et al. [31], Orlandi et al. [32] performed simulations in channels with transverse ribs (square, triangular and circular) and regular roughness (cubes) with focus on augmenting heat transfer through passive means. These studies confirmed that the 
roughness increases heat transfer less than drag, and proposed that the pressure-temperature gradient correlation is important in maintaining the turbulent heat flux. They also point out that the turbulent fluxes are usually the largest component of the total flux. Nagano et al. [30] also determined that k-type roughness resulted in better heat transfer performance compared with transverse-rib and d-type roughness. Leonardi et al. [31] deduced that the heat transfer augmentation from roughness is mostly due to ejections at the leading edge of the roughness elements. They note that the spacing between roughness elements has a crucial influence on heat transfer and mixing since complete reattachment ahead of a downstream roughness element results in a strong stagnation at the leading edge, which results in stronger ejections. They show that scalar fluxes adhere to Townsend's similarity away from the crests (distance depends on the geometry) and that turbulent Prandtl number also collapses on the smooth wall case away from the wall. In the roughness layer, however, the turbulent Prandtl number can be higher or lower than the smooth-wall one, depending on the geometry and flow conditions. They also considered the dispersive heat fluxes, observing that the maximum dispersive flux is located at crests plane and an additional, smaller peak is present above the crest plane. The relative contribution of the dispersive fluxes was found to depend on the geometry and flow conditions, but not definitive conclusion was made on the issue. This was confirmed by Li and Bou-Zeid [33], who also stated that the dispersive stresses and scalar fluxes show more pronounced differences compared with their turbulent counterparts, suggesting that they are due to the non-local action of pressure. They also highlighted the need for further examination of the dispersive terms in the context of passive scalar trnasport.

Recently, Forooghi et al. 34, 35] performed a systematic study of the effects of roughness morphology (geometry, density, solidity, etc.) with artificial and realistic surfaces. They point out that the "sheltering" effect behind roughness elements reduces the Stanton number for denser roughness and that the roughness geometry can affect scalar and momentum differently. MacDonald et al. [36] studied channels with wavy walls, and presented a correlation between the shift in the logarithmic profile, or roughness function, of passive scalar versus the equivalent sand-grain size $k_{s}$. They quantified the scalar roughness function and concluded that it tends asymptotically to a value of $\sim 4.4$ as $k_{s}$ increases, unlike the velocity roughness functions that increases monotonically. For $\operatorname{Pr}=0.7$, they found that the scalar roughness function was smaller than the velocity counterpart. They also illustrated 
the "sheltering" effect and how the instantaneous scalar forms a diffusive sublayer that remains attached to the surface, even at high Reynolds numbers (as opposed to the velocity, which separates between roughness elements). Peeters and Sandham [37], using grit-blasted surfaces, also quantified the scalar roughness function, confirming the trend shown by [36]. They further considered the Reynolds-Analogy ratio, and the behaviour of the scalar streaks, compared the Reynolds stresses and scalar fluxes and performed Quadrant Analysis on the wall-normal turbulent stresses and fluxes. They concluded that within the roughness the dominant events (quadrants) of the turbulent scalar flux are different than those of the turbulent stresses while above the roughness they match. Both works identified that in the fully rough regime, the scalar exhibits a thin thermal diffusive sublayer which closely follows the geometry, resembling the viscous sublayer on smooth wall. The treatment of scalar variance budget in rough-wall flows, particularly w.r.t form-induced terms, received little-to-no attention. Specifically, to the best of the authors' knowledge, the form-induced contribution to the scalar variance production was not investigated at all. This is in contrast to works such as Yuan and Aghaei Jouybari [38] which covered the form-induced terms in the momentum variance budgets extensively, indicating that form-induced terms are important in momentum balance and budgets.

The purpose of the present paper is to examine the mechanisms that drive the exchange of scalar in the roughness sublayer, comparing them with those that govern the momentum transfer. Special focus is given to the budgets of scalar variances and Reynolds stresses, in particular w.r.t the form-induced terms in the momentum and energy balance, and highlight their effect on the different behaviours of streamwise velocity and scalar. This is achieved by performing direct numerical simulations of the flow in a plane channel with rough walls. The problem will be formulated in the next Section. Then, some results will be presented; concluding remarks will close the paper.

\section{PROBLEM FORMULATION}

We consider the transport of a passive scalar in a fully-developed turbulent channel with rough walls, illustrated in Figure 1. The flow is driven by a mean streamwise pressure gradient in a periodic domain. A constant flux of the passive scalar is maintained through the walls, which is a good approximation of many practical applications, and was considered 


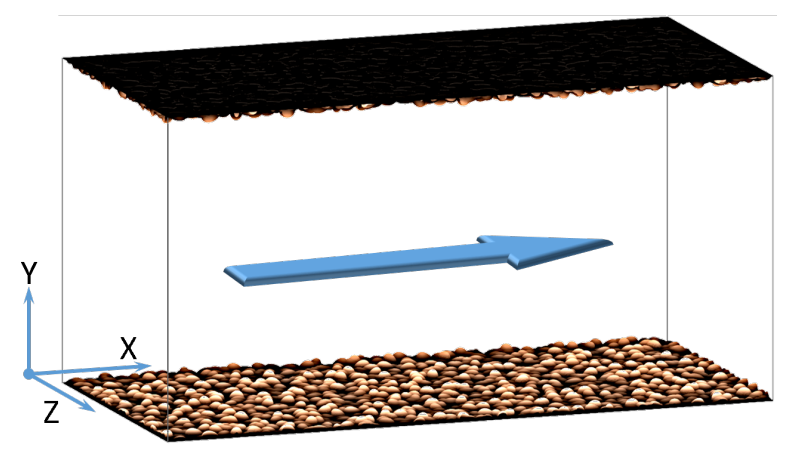

(a)

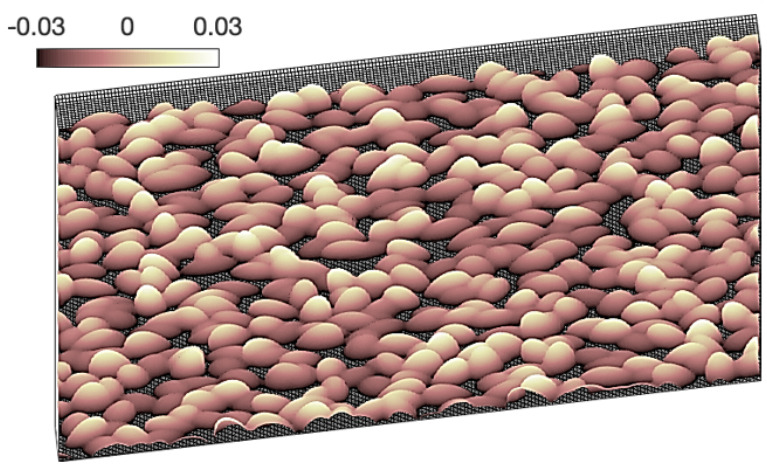

(b)

FIG. 1: (a) Sketch of the computational domain. The arrow indicates the flow direction. (b) Detail of the roughness geometry and mesh size. The colour indicates the wall-normal (y) distance relative to the virtual wall (to be defined in Section III B.

in previous works [23, 24, 29, 37].

The transport of momentum is governed by the incompressible Navier-Stokes equations and mass conservation:

$$
\begin{aligned}
\frac{\partial u_{k}}{\partial x_{k}} & =0 \\
\frac{\partial u_{i}}{\partial t}+\frac{\partial\left(u_{i} u_{k}\right)}{\partial x_{k}} & =\Pi \delta_{i 1}-\frac{\partial p}{\partial x_{i}}+\nu \nabla^{2} u_{i}+F_{i}
\end{aligned}
$$

where $i=1,2$, and 3 (or $x, y$, and $z$ ) are the Cartesian coordinates in the streamwise, wallnormal and spanwise directions respectively. $u_{i}$ (or $u, v$ and $w$ ) are the velocity components in the Cartesian directions, $p=P / \rho$ is the pressure divided by the (constant) density, $F_{i}$ is a forcing term used by the Immersed Boundary Method (IBM) to impose the roughness geometry in the Cartesian domain. $\Pi$ is the driving pressure gradient, which is adjusted at each time to maintain a constant mass flux through the channel. No-slip conditions are used at the wall, while periodicity is enforced in the streamwise and spanwise directions.

For the passive scalar, we follow the approach of [23], which has been used extensively in the literature. Note that, although this is valid for any passive scalar, for brevity we will often refer to it as the temperature, and use the corresponding nomenclature (Prandtl number, specific heat etc). Starting with the passive scalar $T$, we define the transformed scalar $\theta \equiv T_{w}-T$, where $T_{w}$ is the time-averaged value of the scalar $T$ at the wall. If a constant flux at the walls is assumed, $T_{w}$ increases linearly with $x$, resulting in the governing 
equation for $\theta$ :

$$
\frac{\partial \theta}{\partial t}+\frac{\partial\left(u_{k} \theta\right)}{\partial x_{k}}=\alpha \nabla^{2} \theta+Q+F_{\theta}
$$

where $\alpha$ is the kinematic diffusivity, and the forcing term $Q$ is

$$
Q=u \frac{d T_{w}}{d x}=\frac{q_{w}}{\rho c_{p} \delta} \frac{u}{U_{b}}
$$

where $c_{p}$ is the specific scalar capacitance (i.e., specific heat, specific moisture etc.). The source term $Q$ in Eq. (4) is defined by the prescribed scalar flux through the wall, $q_{w}$. The ratio of viscosity to diffusivity defines the Prandtl number $\operatorname{Pr}=\nu / \alpha$. We considered $\operatorname{Pr}=0.7-1.41$, the range for which the Reynolds analogy is known to hold. Using this formulation, $\theta$ also satisfies periodic boundary conditions in $x$ and $z$, while a Dirichlet boundary condition, $\theta=0$, can be applied at the wall; this implies that the wall fluctuations are zero, while a constant wall flux allows for wall fluctuation. Kasagi et al. [39] showed that for this range of Prandtl number the fluctuations at the wall are small enough to justify this assumption. This is further supported by the findings of later works which used different approaches to maintain a constant wall flux without limiting wall fluctuation [25, 26, 29].

The roughness is modelled by an Immersed-Boundary Method (IBM) based on the Volume-of-Fluid (VoF) approach [40]. The surface geometry is a distribution of randomly oriented ellipsoids with semiaxes $k, 1.4 k$ and $2 k$ where $k$ is $4 \%$ of the effective channel halfheight. The roughness crest is located at $y_{c r} \approx 1.5 k \approx 0.06 \delta$. This model results in $k$-type roughness, in which the equivalent sand-grain roughness $k_{s} \simeq k$ in the transitionally rough regime, and $k_{s} \simeq 1.6 k$ in fully rough flow.

The governing equations are solved using second-order accurate central difference in space on a staggered mesh. A fractional-step method is used for the time advancement [41, 42]. A second-order accurate semi-implicit time advancement method is employed in which the Crank-Nicolson scheme is used for the wall-normal diffusive terms, while low-storage thirdorder Runge-Kutta scheme is applied to the remaining terms. The Poisson equation is solved directly using an efficient Fourier Transform solver. The code is parallelized using the Message-Passing Interface (MPI). The numerical model has been widely validated for rough-wall flows [43, 44].

The channel half-width, $\delta$ and the bulk velocity $U_{b}$, can be used to define the bulk Reynolds number $R e_{b}=U_{b} \delta / \nu$. All calculations are performed with $R e_{b}=21,000$, resulting in a Reynolds number based on friction velocity $u_{\tau}$ of $R e_{\tau} \simeq 1,020$ for the smooth-wall case 
TABLE I: Summary of the numerical setup.

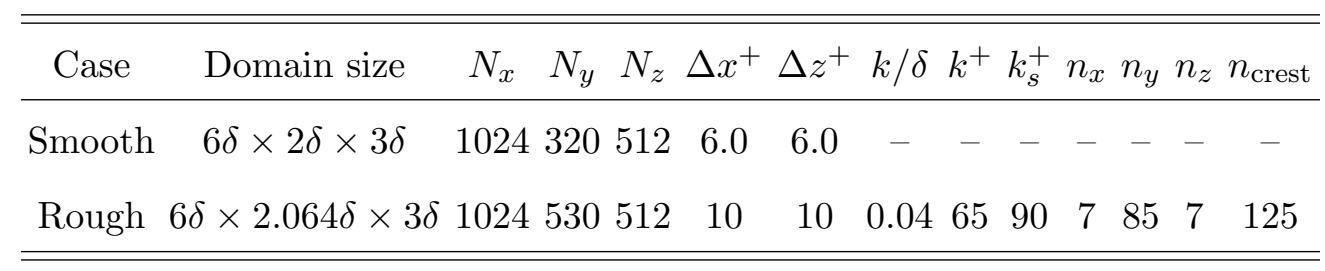

and $R e_{\tau} \simeq 1,700$ for the rough-wall one. In calculations of channel flow, one can maintain either a constant $R e_{b}$, or a constant $R e_{\tau}$; both approaches can be found in the literature. We chose a constant $R e_{b}$ to make our results more directly applicable to boundary layers, in which the free-stream velocity (rather than the friction velocity) is constant. The equivalent sandgrain height, in wall units, is 90 , so that the flow is close to the fully rough regime, as will be further discussed later. For the smooth case, $1024 \times 320 \times 512$ grid points are used to discretize a domain of dimensions $6 \delta \times 2 \delta \times 3 \delta$, and $1024 \times 530 \times 512$ are employed in the rough one. Note that in the latter, the actual domain height is $2.064 \delta$ (rather than $2 \delta$ ) to compensate for the blockage due to the roughness. This results in $\Delta x^{+}=\Delta z^{+} \simeq 6$ in the smooth case, and 10 in the rough one. $\Delta z^{+} \simeq 10$ is slightly larger than the value used in most DNS of this type (usually around 6) but the size of the turbulent eddies near the wall, in this flow, is determined by the roughness geometry and not by the viscous sublayer physics. Although this domain is somewhat smaller (in $x$ and $z$ ) than those commonly used, it is significantly larger than the minimal channel used by [36] in similar calculations. The resolution of the roughness is $n_{x}=n_{z}=7$ cells per $k$ in the $x$ and $z$ directions while in the $y$ direction there are $n_{y}=85$ cells per $k$ and a total of $n_{\text {crest }}=125$ cells covering the region from the base to the crest. Yuan and Piomelli [45] showed that, as long as at least four points are used in each wall-parallel direction, the drag and the flow statistics converge. A grid-refinement study, reported in Appendix $\mathrm{A}$, indicates that this resolution is sufficient for all Prandtl numbers. The parameters of the calculations are summarized in Table 1 . 


\section{RESULTS}

\section{A. Triple Decomposition}

Several averaging operators can be used in rough-wall channel flows [46]. Time averaging is indicated by an overbar. In the $x z$-plane we utilize two spatial averages as defined by [46]: the intrinsic spatial average $\langle\cdot\rangle$, and the superficial average $\langle\cdot\rangle_{s}$. The whole plane surface is denoted by $A_{0}$ while $A_{f}$ denotes the region of the plane surface occupied by fluid. The fluid fraction $\phi(y)=A_{f} / A_{0}$, i.e., the ratio of fluid area to total area, relates the two averages: $\langle\theta\rangle_{s}=\phi\langle\theta\rangle$. The spatial averages do not commute with spatial derivatives, resulting in additional terms in the transport equations [46, 47], which will be discussed in section III] in their respective context.

A flow quantity $f$ can be decomposed into three terms [46 48]: (1) a component that is averaged in time and space (or double-averaged), $\langle\bar{f}\rangle ;(2)$ the spatial variation of the time-averaged quantity, $\widetilde{f}$; and (3) the instantaneous fluctuation $f^{\prime}$ :

$$
f\left(x_{i}, t\right)=\bar{f}\left(x_{i}\right)+f^{\prime}\left(x_{i}, t\right)=\langle\bar{f}\rangle(y)+\tilde{f}\left(x_{i}\right)+f^{\prime}\left(x_{i}, t\right) .
$$

$\widetilde{f}$ is known as the "wake field", or "form-induced perturbation". It represents the deviation of the time-averaged field from the time-and-space averaged one, and highlights the geometryinduced effects. By definition, $\langle\tilde{\cdot}\rangle=0 ; \stackrel{\sim}{*}=0$ in the smooth case and away from the roughness sublayer, where the time-averaged fields are spatially uniform (in $x$ and $z$ ), and the triple decomposition reverts to the standard Reynolds decomposition. The wake-field is a (temporally) persistent spatial perturbations field, i.e., it is non-zero whenever the time averaged field is non-uniform.

\section{B. Mean velocity and scalar profiles}

Figure 2 shows profiles of the temporally and superficially averaged streamwise velocity $\langle\bar{u}\rangle_{s}$ and scalar $\langle\bar{\theta}\rangle_{s}$. The normal coordinate $y$ is displaced by the location of the force centroid $d$ [49]. To define wall units, velocities are normalized by $u_{\tau}=\left(\tau_{w} / \rho\right)^{1 / 2}$, lengths by $\nu / u_{\tau}$, and the scalar by $\theta_{\tau}=q_{w} /\left(\rho c_{p} u_{\tau}\right)$.

A logarithmic layer is present for the scalar, similar to the one for the velocity, with a slightly lower slope, as also observed by other researchers [29, 36]. While the Von Kàrmàn 

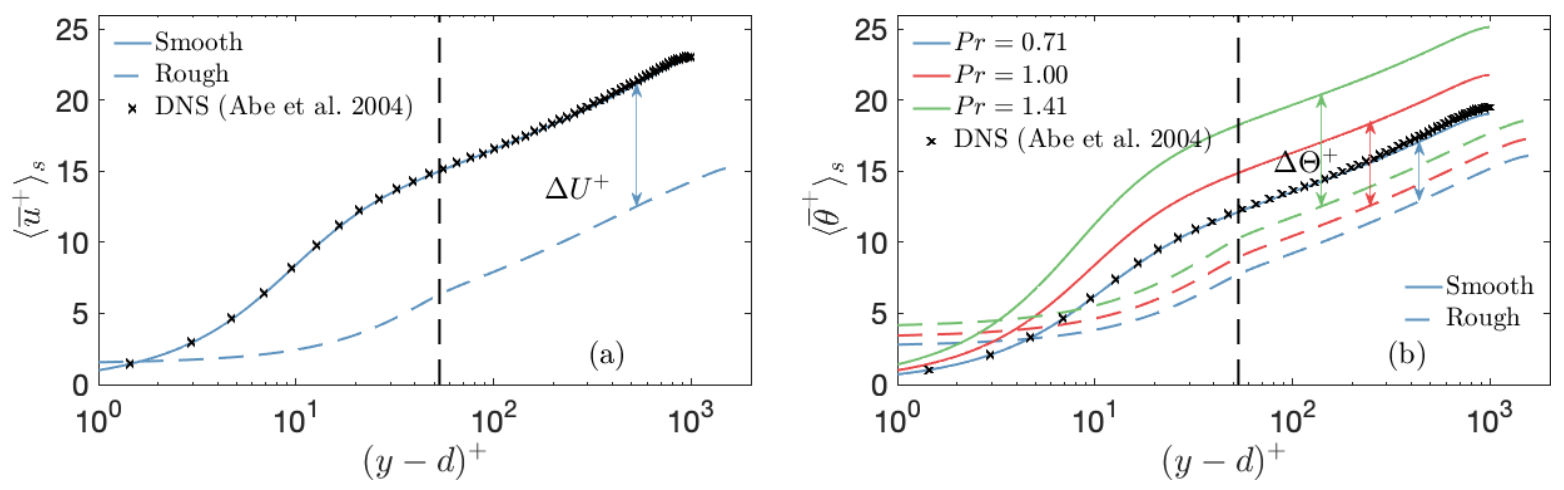

FIG. 2: Double-averaged velocity (a) and scalar (b). The dashed vertical line denotes the roughness crest. $\times$ : DNS data by Abe et al. [26].

constant of the scalar logarithmic layer $\left(\kappa_{\theta}=0.46\right)$ is independent of the Prandtl number, the intercept is not, increasing with $\mathrm{Pr}$. The data is in good agreement with the DNS by Abe et al. [26].

When the wall is rough, the logarithmic layers for velocity and scalar maintain the same slope, but are shifted downwards; this offset is known as the "roughness function". An extensive discussion on the roughness function in this context can be found in Peeters and Sandham [37], from which the definitions for the roughness functions are taken:

$$
\Delta U^{+}=\left.\langle\bar{u}\rangle_{s}^{+}\right|_{k_{s}=0}-\left.\langle\bar{u}\rangle_{s}^{+}\right|_{k_{s}} ; \quad \Delta \Theta^{+}=\left.\langle\bar{\theta}\rangle_{s}^{+}\right|_{k_{s}=0}-\left.\langle\bar{\theta}\rangle_{s}^{+}\right|_{k_{s}}
$$

Here, the roughness function is calculated by averaging the difference between the log law on a region over which they are nearly constant. We used $200<y^{+}<600$; the results are not strongly dependent on the choice of the averaging interval, unless it extends into the wake region or the buffer layer, nor on the choice of origin (force centroid or geometric mean). The roughness function for the velocity, $\Delta U^{+}$, is in good agreement with that obtained by other researchers at comparable equivalent sandgrain roughnesses, Figure 3(a) and Table II, while the values of $\Delta \Theta^{+}$predicted in the present study are consistently higher, perhaps because of the different geometries, that have some effect at the lower edge of the fully rough regime. The value of $k_{s}^{+}=100$ is at the beginning of the fully rough regime, where $\Delta U^{+}$becomes independent of the type of roughness, and follows a logarithmic behaviour [11], and the drag is mostly due to the pressure drag. Some residual transitional effect can still be observed, however, in the difference between the various values obtained with different roughness shapes. The roughness function of the scalar, $\Delta \Theta^{+}$, depends on the Prandtl number: the 
TABLE II: Summary of the mean velocity and scalar profile parameters.

\begin{tabular}{ccccccc}
\hline \hline & $R e_{\tau}$ & $k_{s}^{+}$ & $\operatorname{Pr}$ & $\Delta \Theta^{+}$ & $\Delta U^{+}$ & Coordinate origin \\
\hline \multirow{2}{*}{ MacDonald et al. [36] } & 1680 & 380 & & 4.3 & 10.9 & \\
& 395 & 90 & & 3.0 & 6.5 & Geometric mean \\
\hline \multirow{2}{*}{ Peeters and Sandham [37] } & 720 & 102 & & 4.7 & 8.6 & \\
& 540 & 80 & 1.0 & 4.6 & 7.7 & Geometric mean \\
\hline \multirow{2}{*}{ Present study } & & & 0.71 & 4.2 & & \\
& 1680 & 90 & 1.0 & 5.7 & 8.6 & Force centroid \\
\hline \hline
\end{tabular}
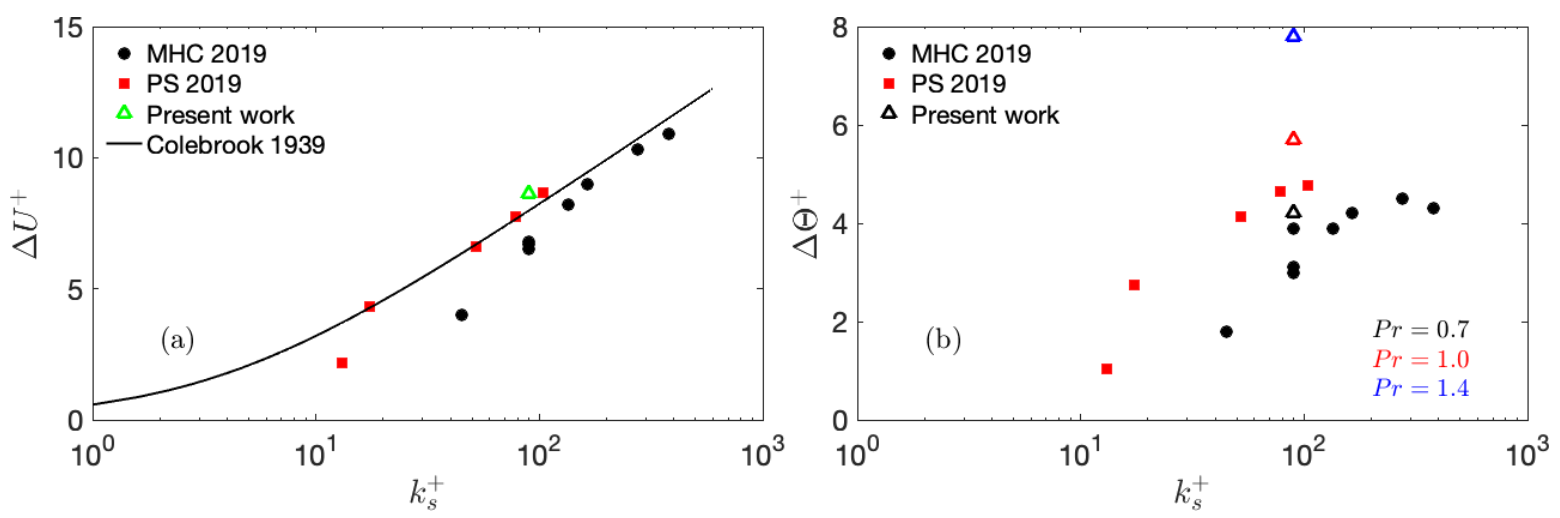

FIG. 3: (a) Velocity and (b) scalar roughness functions. Comparison data taken from MacDonald et al. [36] (MHC 2019) and Peeters and Sandham [37] (PS 2019)

logarithmic layers for the scalar in the rough-wall case are more clustered together compared to the smooth-wall case, resulting in the decrease of $\Delta \Theta^{+}$as $\operatorname{Pr}$ decreases. $\Delta \Theta^{+}$in this range of $\operatorname{Pr}$ is lower than the velocity roughness function, $\Delta U^{+}$, in line with the findings of [36, 37].

\section{Mean momentum and scalar balances}

Applying the double-averaging procedure to the conservation of streamwise momentum, (2), yields the mean momentum balance:

$$
-\langle\bar{\Pi}\rangle_{s}=\frac{d}{d y}\left[\nu \frac{d\langle\bar{u}\rangle_{s}}{d y}-\left\langle\overline{u^{\prime} v^{\prime}}\right\rangle_{s}-\langle\widetilde{u} \widetilde{v}\rangle_{s}\right]+f_{p}+f_{\nu}
$$


where the LHS is the driving mean pressure gradient; on the RHS, in addition to the gradients of viscous and stochastic shear stresses, which are also present in smooth-wall flows, there are three terms that are directly induced by the roughness: the gradient of the dispersive stress $\langle\widetilde{u} \widetilde{v}\rangle_{s}$, the form-induced pressure force $f_{p}$, and the form-induced viscous force $f_{\nu}$. The last two are given by:

$$
f_{p}=-\left\langle\frac{\partial \widetilde{p}}{\partial x}\right\rangle_{s} ; \quad f_{\nu}=\left\langle\frac{\partial}{\partial x_{k}}\left(\nu \frac{\partial \widetilde{u}}{\partial x_{k}}\right)\right\rangle_{s}-2 \nu \frac{d\langle\bar{u}\rangle}{d y} \frac{d \phi}{d y}-\nu\langle\bar{u}\rangle \frac{d^{2} \phi}{d y^{2}}
$$

Similarly, double-averaging Eq. (3) yields

$$
-\langle\bar{Q}\rangle_{s}=\frac{d}{d y}\left[\nu \frac{d\langle\bar{\theta}\rangle_{s}}{d y}-\left\langle\overline{\theta^{\prime} v^{\prime}}\right\rangle_{s}-\langle\widetilde{\theta} \widetilde{v}\rangle_{s}\right]+f_{\alpha}
$$

where the LHS is the averaged scalar source term $Q$ given in Eq. (4) and on the RHS the first two terms are the diffusive and turbulent fluxes, which are also present in smooth-wall channel, while the last two terms are the dispersive flux and the form-induced conduction, $f_{\alpha}$, which is the equivalent of $f_{\nu}$ :

$$
f_{\alpha}=\left\langle\frac{\partial}{\partial x_{k}}\left(\alpha \frac{\partial \tilde{\theta}}{\partial x_{k}}\right)\right\rangle_{s}-2 \alpha \frac{d\langle\bar{\theta}\rangle}{d y} \frac{d \phi}{d y}-\alpha\langle\bar{\theta}\rangle \frac{d^{2} \phi}{d y^{2}}
$$

Integrating Eq. (7) and (9) in the wall-normal direction results in the total stress and scalar flux. The form-induced contributions are:

$$
F_{p}=\int f_{p} d y ; \quad F_{\nu}=\int f_{\nu} d y ; \quad F_{\alpha}=\int f_{\alpha} d y
$$

Figure 4 shows the contribution of the various terms to the total stress and flux. Note that the total stress is not linear in the roughness sublayer because the fluid fraction is not constant. The total drag acting on a rough-wall is the sum of viscous and pressure contributions [9, 10, 47]. The latter, also known as form drag, is a result of the pressure difference between the stagnation regions in front of the roughness elements and the separated-flow areas downstream of them. It is dominant in the fully rough regime. In the present case, $70 \%$ of the total wall stress is due to the form drag, $F_{p}$; since the flow is at the lower edge of the fully rough regime, the viscous drag is significantly smaller, but not negligible, contributing the remaining $30 \%$ as form-induced viscous force. The form-induced terms $\left(F_{p}, F_{\nu}\right.$, and $\left.\langle\widetilde{u} \widetilde{v}\rangle_{s}\right)$ account for more than half of the momentum flux up to the virtual wall, $y=d$; 

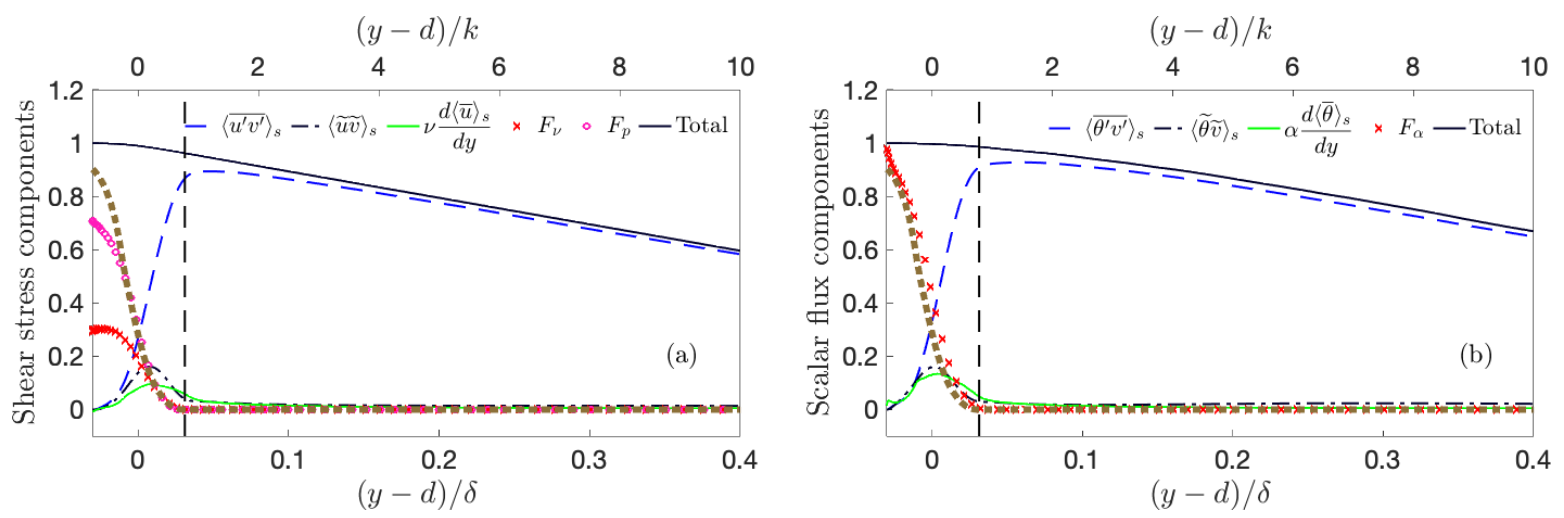

FIG. 4: Mean (a) stresses and (b) scalar fluxes $(\operatorname{Pr}=1.0)$. Stress and flux components are in wall units. The dashed vertical line marks the roughness crest; the thick brown dotted line is the solid fraction $1-\phi$.

from this point on the stochastic Reynolds stresses dominate the momentum transfer. The dispersive stresses, however, are not insignificant even near the crest.

Dipprey and Sabersky [19] showed experimentally, in the context of heat transfer, that the behaviour of a scalar quantity in the presence of roughness is different than that of momentum, specifically pointing to the absence of a pressure or pressure-like mechanism. Figure 4(b) shows that the scalar wall flux is indeed almost entirely due to the form-induced conductive flux $\left(F_{\alpha}\right)$. The form-induced flux follows very closely the distribution of the solid fraction, $1-\phi$, emphasizing the importance of the wetted area to scalar transport, while for momentum the viscous contribution is overall much less significant, due to the pressure dominance. The behaviour of the dispersive scalar flux $\langle\widetilde{\theta} \widetilde{v}\rangle_{s}$ is similar to that observed for $\langle\widetilde{u} \widetilde{v}\rangle_{s}$.

\section{Turbulent stresses and fluxes}

Figures 5.6 show the second-order moments of velocity and scalar for $\operatorname{Pr}=0.71$ and 1.0. The agreement with the data by [26] at $\operatorname{Pr}=0.71$ is very good. As observed previously [13, 14, 50, 51] the presence of roughness dampens the streamwise fluctuation, $\left\langle\overline{u^{\prime} u^{\prime}}\right\rangle_{s}$, by approximately $50 \%$ while $\left\langle\overline{v^{\prime} v^{\prime}}\right\rangle_{s}$ and $\left\langle\overline{w^{\prime} w^{\prime}}\right\rangle_{s}$ are nearly unchanged, making the Reynoldsstress tensor more isotropic. The scalar variance $\left\langle\overline{\theta^{\prime} \theta^{\prime}}\right\rangle_{s}$ is also reduced, but less than the streamwise flux $\left\langle\overline{\theta^{\prime} u^{\prime}}\right\rangle_{s}$ or streamwise velocity variance $\left\langle\overline{u^{\prime} u^{\prime}}\right\rangle_{s}$, at all Prandtl numbers considered. 

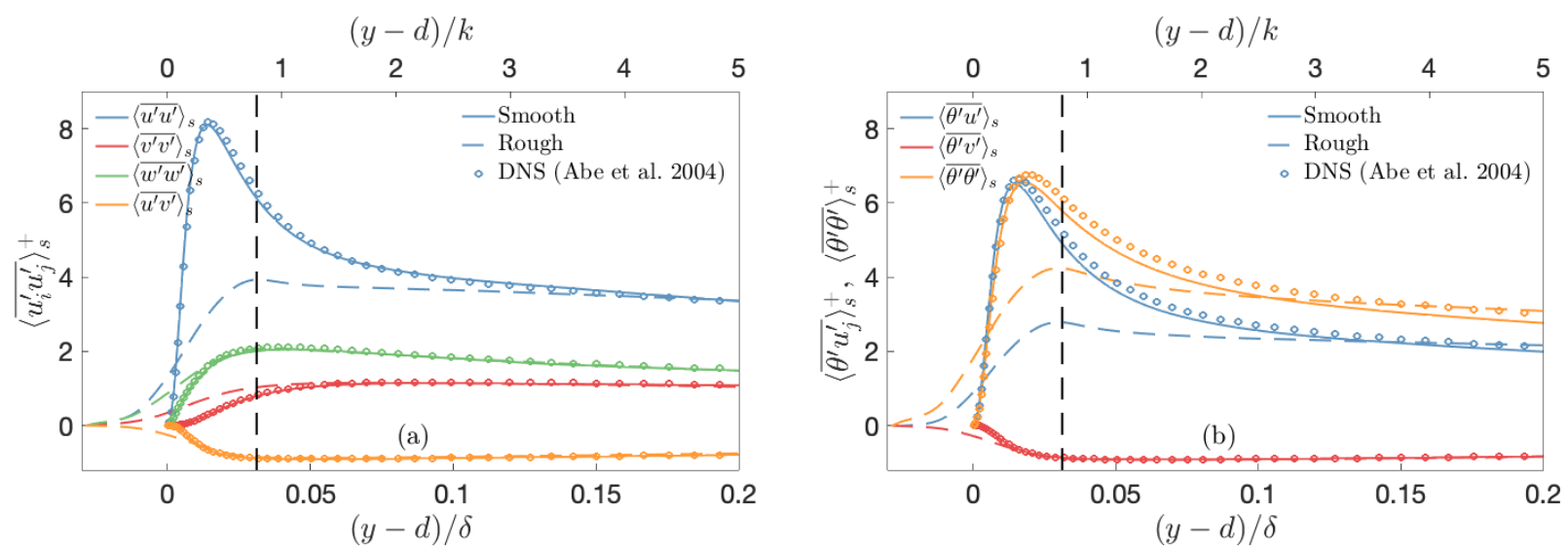

FIG. 5: Superficially averaged Reynolds stresses, scalar fluxes, and scalar variance; $\operatorname{Pr}=0.71$. All moments are normalized by viscous units. (a) Stochastic Reynolds stresses; (b) stochastic scalar fluxes and variance.

$\left\langle\overline{\theta^{\prime} v^{\prime}}\right\rangle_{s}$ and $\left\langle\overline{u^{\prime} v^{\prime}}\right\rangle_{s}$ remain similar, as noted by Peeters and Sandham [37]. All the quantities collapse on their smooth-wall equivalent several roughness-heights from the wall $\left(y / k_{s} \simeq 3-5\right)$, confirming that Townsend's similarity hypothesis, known to apply for momentum [16, 52], also applies for scalar in the range of Prandtl numbers examined. The stochastic fluxes are significant inside the roughness sublayer; in fact, apart for the wallnormal one, all the components have their peak below the roughness crest.

While the dispersive stresses have been examined before [38, 43], very little attention was given to the scalar dispersive fluxes, although they were shown to possibly be significant [31, 33]. The dispersive stresses and fluxes, Figures 6(c,d) are significant. Throughout the roughness sublayer they are of the same order as (and sometimes larger than) the stochastic ones, as can be seen in Figure 7. Note that deep into the roughness sublayer $(y / k<0.2)$ both the stochastic and dispersive fluxes, $\left\langle\overline{\theta^{\prime} u^{\prime}}\right\rangle_{s}$ and $\langle\widetilde{\theta} \widetilde{u}\rangle_{s}$ are very small and negative, and they change sign at different points; this causes the spike in Figure 7. The dispersive fluxes contribute a larger percentage of the scalar transport, compared to the dispersive stresses. Above the roughness sublayer the dispersive stresses and fluxes are small, but not zero, as they should be. Coceal et al. [53], Leonardi et al. [31], Yuan and Piomelli [44] and Yuan and Aghaei Jouybari [38] also observed similar phenomena. Since the time scales outside the roughness are significantly longer than those of the near-wall region, an extremely long averaging time is required for the the point-wise time-averaged quantities in the bulk region to converge to the correct, spatially-homogeneous ensemble average. 

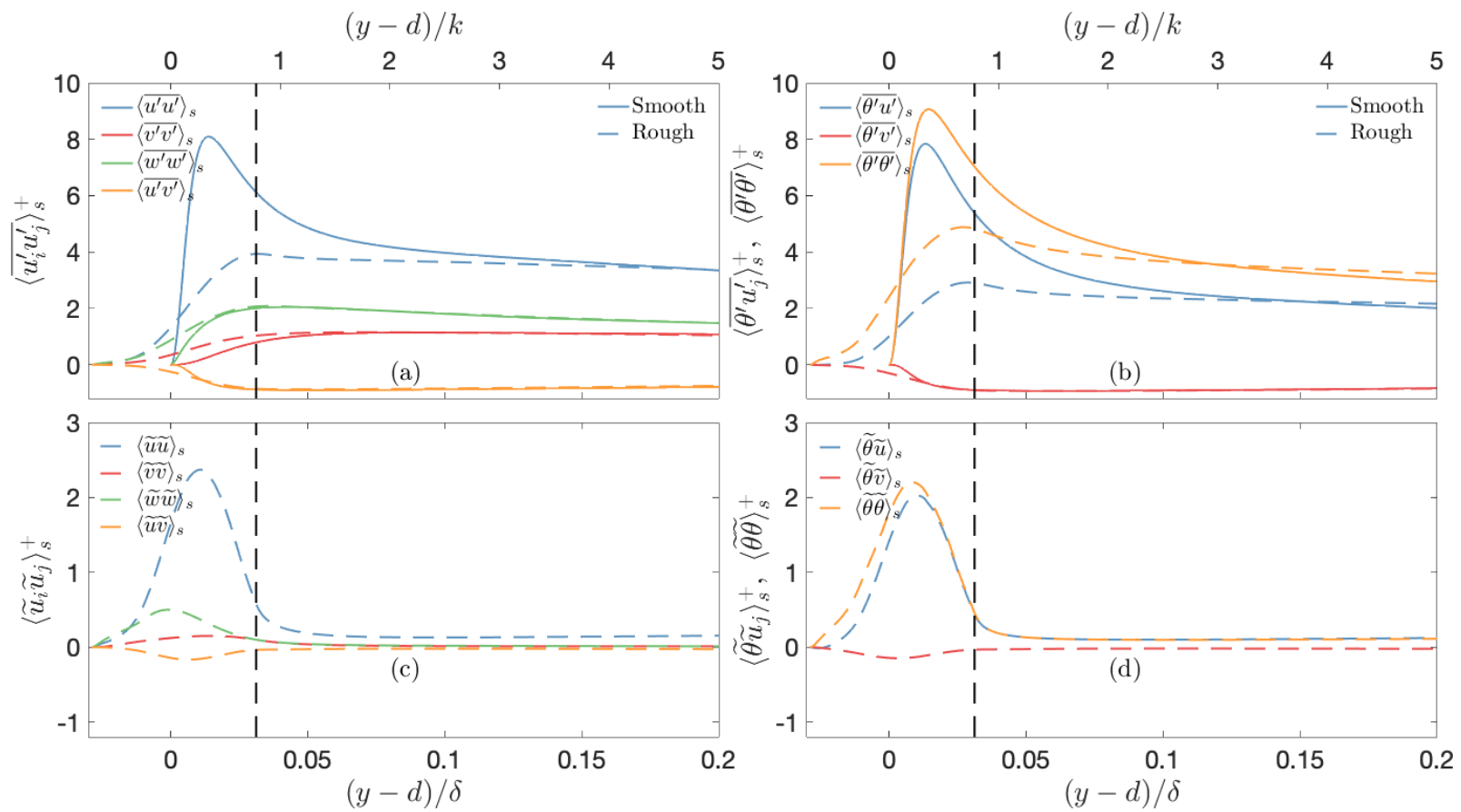

FIG. 6: Superficially averaged Reynolds stresses, scalar fluxes, and scalar variance; $\operatorname{Pr}=1.00$. All moments are in normalized by viscous units. (a) Stochastic Reynolds stresses; (b) stochastic scalar fluxes and variance; (c) dispersive stresses; (d) dispersive scalar fluxes and variance.
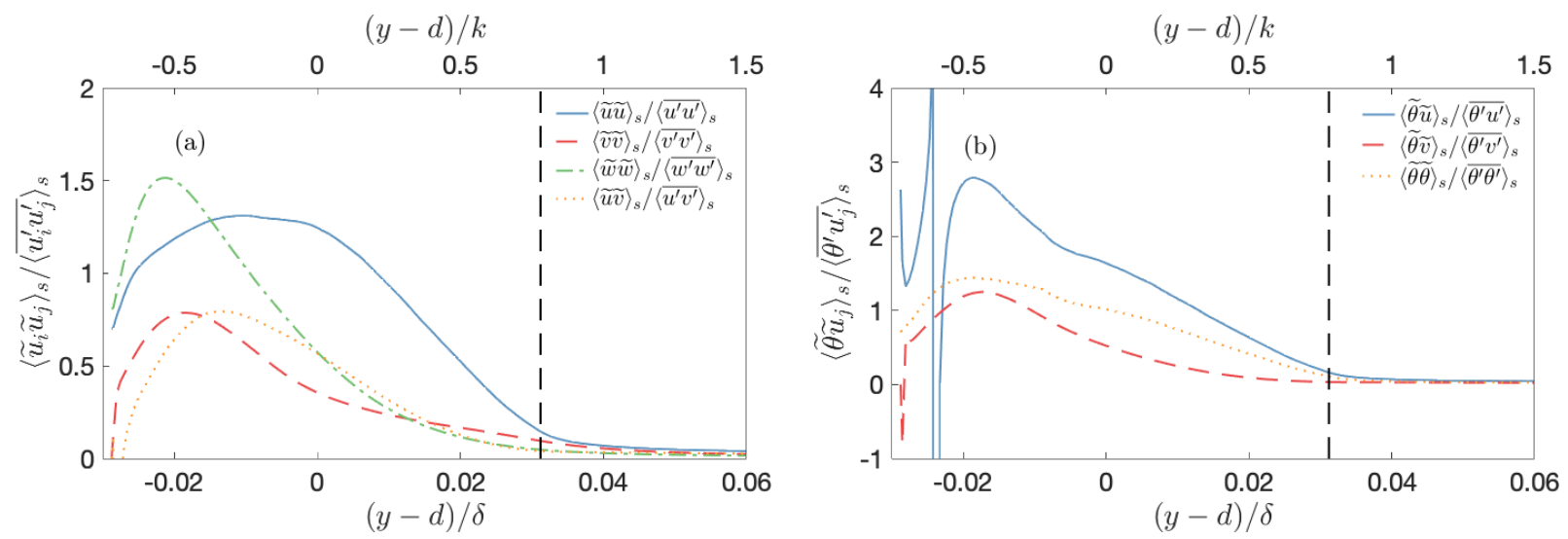

FIG. 7: Ratio of the superficially averaged dispersive stresses and scalar fluxes to their stochastic counterpart; $\operatorname{Pr}=1.00$. (a) Reynolds stresses; (b) scalar fluxes and variance. 


\section{E. Reynolds stress and scalar variance budgets}

The budgets of the Reynolds stresses and scalar fluxes can be written as:

$$
\begin{aligned}
& 0=\mathcal{P}_{i j}+\mathcal{A}_{i j}+\mathcal{T}_{i j}+\mathcal{D}_{i j}-\varepsilon_{i j}+\Pi_{i j} \\
& 0=\mathcal{P}_{\theta}+\mathcal{A}_{\theta}+\mathcal{T}_{\theta}+\mathcal{D}_{\theta}-\varepsilon_{\theta}+Q_{\theta}
\end{aligned}
$$

where the terms on the RHS of these quations are the production $\mathcal{P}$, wake transport $\mathcal{A}$, turbulent transport $\mathcal{T}$, viscous/conductive diffusion $\mathcal{D}$ and dissipation $\varepsilon$. In addition, Eq. (12) contains the pressure-work term $\Pi_{i j}$ (not to be confused with $\Pi$ in Eq. (2)) and Eq. (13) contains the source term $Q_{\theta}$ (not to be confused with $Q$ in Eq. (3)). The explicit expressions of the terms and additional details can be found in Appendix $B$.

Figure 8 shows the budgets of the streamwise normal Reynolds stress, $\left\langle\overline{u^{\prime} u^{\prime}}\right\rangle_{s}$, and scalar variance $\left\langle\overline{\theta^{\prime} \theta^{\prime}}\right\rangle_{s}$, normalized by $u_{\tau}, \theta_{\tau}$ and $\nu$. The scalar is shown for $\operatorname{Pr}=1.0$ in order to emphasize the difference between the quantities under the most similar conditions.

Of particular interest is the production $\mathcal{P}$, which can be decomposed into "shear production", $\mathcal{P}^{s}$ and "form-induced production", $\mathcal{P}^{f i}$. The former is present in both smooth and rough flows, whereas the latter is due to the roughness geometry. The form-induced production can be further split into the "mean production" $\mathcal{P}^{m}$, and the "wake production", $\mathcal{P}^{w}$ :

$$
\begin{gathered}
\mathcal{P}_{11}=\underbrace{-2\left\langle\overline{u^{\prime} v^{\prime}}\right\rangle_{s} \frac{d\langle\bar{u}\rangle}{d y}}_{\mathcal{P}_{11}^{s}} \underbrace{-2\left\langle\overline{u^{\prime} u_{k}^{\prime}}\right\rangle_{s}\left\langle\frac{\partial \widetilde{u}}{\partial x_{k}}\right\rangle}_{\mathcal{P}_{11}^{m}} \underbrace{-2\left\langle\widetilde{u^{\prime} u_{k}^{\prime}} \frac{\partial \widetilde{u}}{\partial x_{k}}\right\rangle_{s}}_{\mathcal{P}_{\theta}^{s}} . \\
\mathcal{P}_{\theta}=\underbrace{-2\left\langle\overline{\theta^{\prime} v^{\prime}}\right\rangle_{s} \frac{d\langle\bar{\theta}\rangle}{d y}}_{\mathcal{P}_{11}^{w}} \underbrace{-2\left\langle\overline{\theta^{\prime} u_{k}^{\prime}}\right\rangle_{s}\left\langle\frac{\partial \widetilde{\theta}}{\partial x_{k}}\right\rangle}_{\mathcal{P}_{\theta}^{m}} \underbrace{-2\left\langle\widetilde{\theta^{\prime} u_{k}^{\prime}} \frac{\partial \tilde{\theta}}{\partial x_{k}}\right\rangle_{s}}_{\mathcal{P}_{\theta}^{w}} .
\end{gathered}
$$

While the shear production represents the work of the mean Reynolds stresses against the mean (double-averaged) velocity gradient, the form-induced production represents the work of the the mean Reynolds stresses (for the case of $\mathcal{P}^{m}$ ) and of the form-induced Reynolds stresses (for the case of $\mathcal{P}^{w}$ ) against the form-induced velocity fluctuations. Analogous interpretations can be given to the production terms for the scalar variance. It has been shown [8, 38] that, in the context of momentum transport, the form-induced production can be significant and provides an alternative mechanism (the "spectral short-cut") for producing turbulent kinetic energy directly at the roughness scales. 

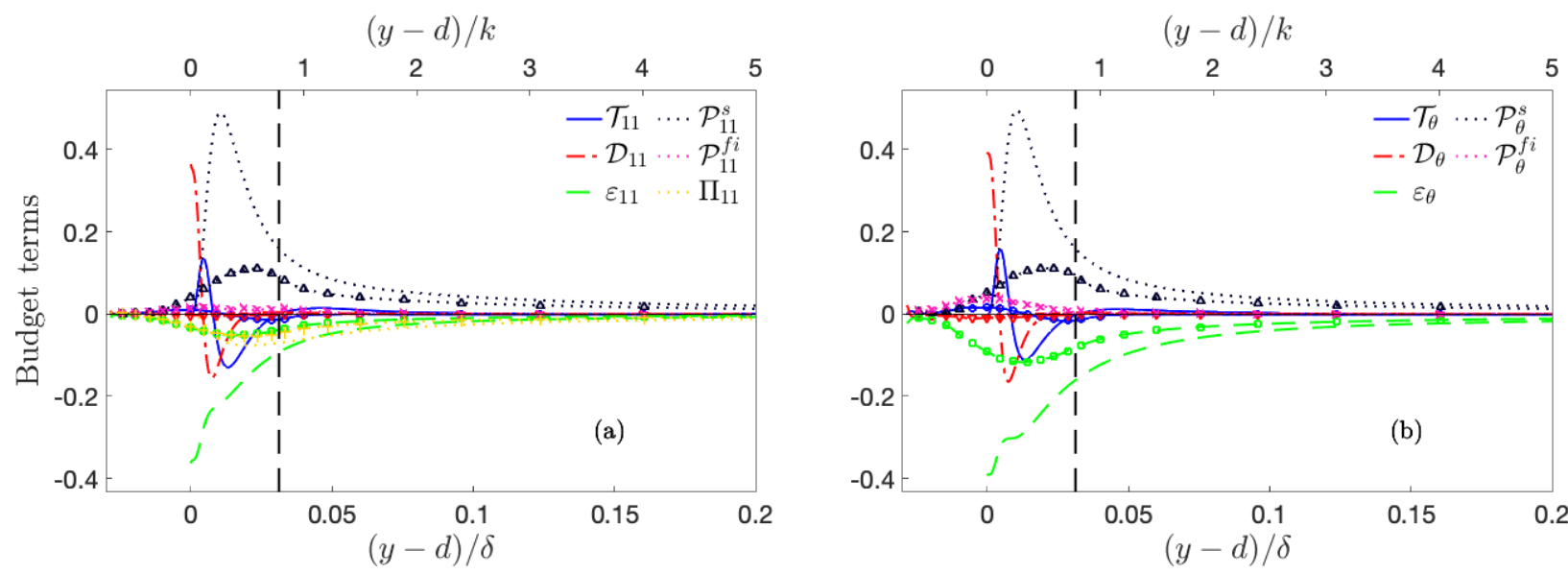

FIG. 8: Budgets of (a) $\left\langle\overline{u^{\prime} u^{\prime}}\right\rangle_{s}$ and $(\mathrm{b})\left\langle\overline{\theta^{\prime} \theta^{\prime}}\right\rangle_{s}$ with $\operatorname{Pr}=1.0$. Lines: Smooth wall; Lines with markers: rough wall. Terms are normalized by $u_{\tau}^{4} / \nu$ in the Reynolds stress budget, and $u_{\tau}^{2} \theta_{\tau}^{2} / \nu$ in the scalar one. The vertical line marks the roughness crest. Only significant terms are shown; enlarged figure including all terms can be found in Appendix B

In the smooth-wall case, the budgets are very similar to each other. The pressure work $\Pi_{11}$, responsible for the energy redistribution between the three normal stresses, acts as a sink term for $\left\langle\overline{u^{\prime} u^{\prime}}\right\rangle_{s}$. Near the wall, $\Pi_{11}$ is not significant compared with $\varepsilon_{11}$, however away from the wall they are roughly equal, and act as the sinks balancing the shear production. In the rough case $\Pi_{11}$ is as significant as $\varepsilon_{11}$ both near and away from the wall, balancing the shear production, which, although much reduced compared to the smooth-wall case, is still the main gain term. All other terms are significantly smaller.

The budget of $\left\langle\overline{\theta^{\prime} \theta^{\prime}}\right\rangle_{s}$ presents some interesting features. An equivalent to $\Pi_{11}$ is not present in the scalar budget while the scalar source term contribution $Q_{\theta}$ is negligible. The dissipation is the leading sink term near the wall, and the only one away from the wall; the magnitude of $\varepsilon_{\theta}$ is, therefore, larger than that of $\varepsilon_{11}$ by an amount roughly equal to $\Pi_{11}$, compensating for the lack of the pressure work. This is consistent in both smooth and rough cases. The shear production $\mathcal{P}_{\theta}^{s}$ is similar to $\mathcal{P}_{11}^{s}$, mirroring the similarity between $\left\langle\overline{u^{\prime} v^{\prime}}\right\rangle_{s}$ and $\left\langle\overline{\theta^{\prime} v^{\prime}}\right\rangle_{s}$ (shown in Figure $6(a, b)$ ) and between the mean velocity and scalar profiles (Figure 2). The form-induced production $\mathcal{P}_{\theta}^{f i}$ is much larger than $\mathcal{P}_{11}^{f i}$. In fact, up to the force centroid, $y=d$, it is the largest positive term in the budget. The larger form-induced production may be a primary reason for the reduced damping of $\left\langle\overline{\theta^{\prime} \theta^{\prime}}\right\rangle_{s}$, compared to $\left\langle\overline{u^{\prime} u^{\prime}}\right\rangle_{s}$, which was mentioned in Section IIID. 

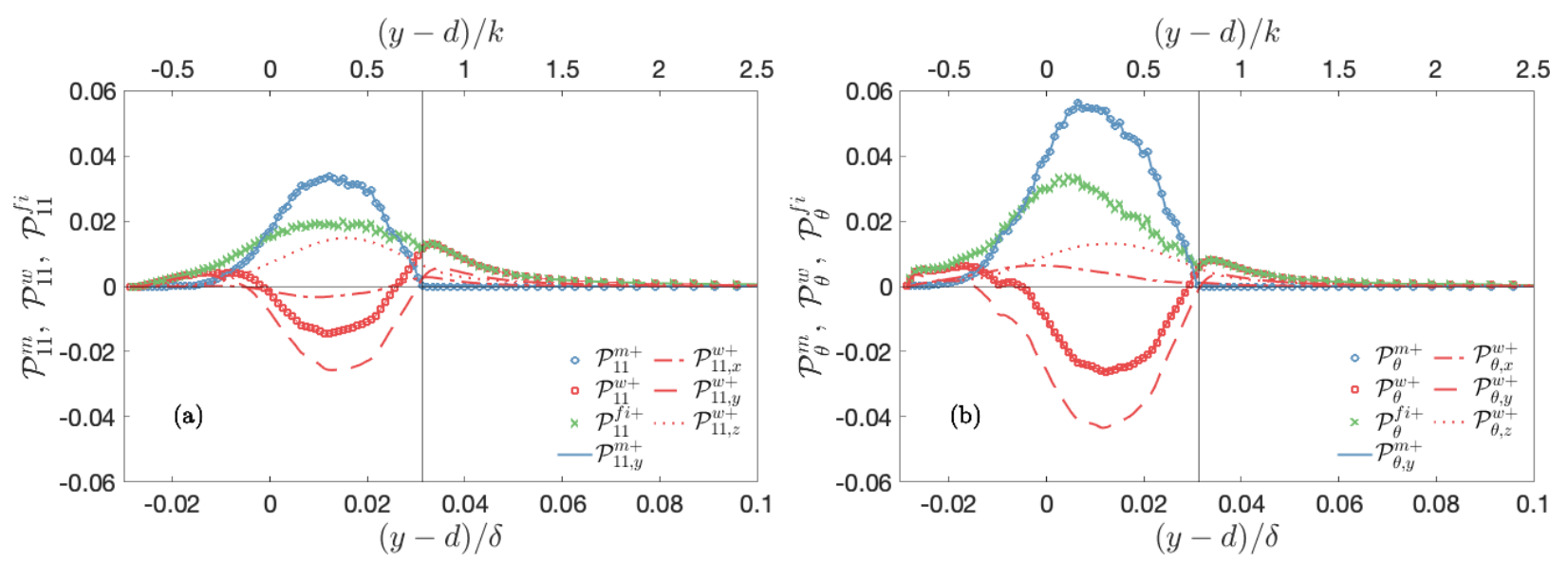

FIG. 9: Breakdown of the contributions to the form-induced production $\mathcal{P}_{11}^{f i}$ and $\mathcal{P}_{\theta}^{f i}$ for $\operatorname{Pr}=1.0$. Terms are normalized by $u_{\tau}^{4} / \nu$ in the Reynolds stress budget, and $u_{\tau}^{2} \theta_{\tau}^{2} / \nu$ in the scalar one. The vertical line marks the roughness crest.

Raupach and Shaw [47] considered the budget of the wake kinetic energy (WKE) for the flow over a canopy, and observed that, if the dispersive stresses are negligible, the WKE equation reduces to

$$
-2\left\langle\widetilde{u^{\prime} u_{k}^{\prime}} \frac{\partial \widetilde{u}}{\partial x_{k}}\right\rangle_{s}=\left\langle\overline{u_{i}}\right\rangle\left\langle\frac{\partial \widetilde{p}}{\partial x_{j}}\right\rangle_{s}
$$

indicating that the wake production represents the work of the mean flow against the pressure drag. In our case, $\mathcal{P}_{11}^{m}$ would also appear on the left-hand-side. This interpretation of the form-induced production does not seem to apply to this case, since the dispersive stresses are not negligible (see Figure 7). Furthermore, the behaviours of $\mathcal{P}_{11}^{f i}$ and $\mathcal{P}_{\theta}^{f i}$ are very similar, despite the fact that there is no pressure term in the scalar equation. For the flow over a rough-wall surface, the WKE balance is considerably more complex than for the canopy. It is then clear that the type of roughness has significant importance over how the two forminduced production components behave and contribute, an issue that could be critical when modelling turbulence.

We next consider the breakdown of the form-induced production into the constituent mean production and wake production. Note that while the shear production only depends on double-averaged quantities, the mean production is a mix of double-averaged and wake quantities and the wake production depends purely on the wake fields. The contributions of these terms can be seen in Figure 9. For both scalar and Reynolds stress the form-induced production is positive, and significantly larger for the scalar than for the Reynolds stress. 
The mean and wake production can be further split into their $x, y$ and $z$ components, and the contribution of each of these terms is also shown in Figure 9. The mean production terms are simpler, as only the $y$-component has a non-zero mean contribution:

$$
\mathcal{P}_{11}^{m}=\mathcal{P}_{11, y}^{m}=-2\left\langle\overline{u^{\prime} v^{\prime}}\right\rangle_{s}\left\langle\frac{\partial \widetilde{u}}{\partial y}\right\rangle ; \quad \mathcal{P}_{\theta}^{m}=\mathcal{P}_{\theta, y}^{m}=-2\left\langle\overline{\theta^{\prime} v^{\prime}}\right\rangle_{s}\left\langle\frac{\partial \widetilde{\theta}}{\partial y}\right\rangle .
$$

For the Reynolds stress, the mean production ( $y$ term) and the $x$-component of the wake production are positive; the mean production nearly balances the $y$-component of the wake production. For the scalar, on the other hand, both $x$ - and $z$-components of the wake production are positive, and combine with the mean production to increase the scalar variance. In both cases, the most important term appears to be the mean production, which, together with the wake production, represents the rate at which energy is transferred from the wake field to the turbulent fluctuations.

The mean production terms $\left(\mathcal{P}_{\theta}^{m}\right.$ and $\left.\mathcal{P}_{11}^{m}\right)$ can be further simplified, given that $\bar{\theta}$ and $\bar{u}$ are zero on the boundary:

$$
\mathcal{P}_{11}^{m}=-2\left\langle\overline{u^{\prime} v^{\prime}}\right\rangle\left(\langle\bar{u}\rangle \frac{d \phi}{d y}\right) ; \quad \mathcal{P}_{\theta}^{m}=-2\left\langle\overline{\theta^{\prime} v^{\prime}}\right\rangle\left(\langle\bar{\theta}\rangle \frac{d \phi}{d y}\right) .
$$

This form underlines a key difference in the roles of the mean and wake production terms: Although all production terms include some indirect effect of the roughness, only $\mathcal{P}^{m}$ depends explicitly on the geometry through the derivative of $\phi$ (and in fact is strictly zero above the crest). The wake production $\mathcal{P}^{w}$, on the other hand, is only related to the geometry indirectly through the adjustment of the mean flow field to the roughness shape (which persists above the roughness crest): upwards jets are generated on the upstream side of the roughness elements, and downwards sweeps on the leeward side, which propagate into the flow above the roughness crest. Note that in roughness geometries in which the fluid fraction $\phi$ is not a function of $y$, (e.g., vegetation canopies, arrays of cubes) the mean production term vanishes [8, 47, 48].

The decomposition (18) also explains why the form-induced production contributes more to $\left\langle\overline{\theta^{\prime} \theta^{\prime}}\right\rangle_{s}$ than to $\left\langle\overline{u^{\prime} u^{\prime}}\right\rangle_{s}$. The mean and wake production terms are mostly opposite in sign. Both mean-production terms are the product of three terms: (1) $\left\langle\overline{u^{\prime} v^{\prime}}\right\rangle$ or $\left\langle\overline{\theta^{\prime} v^{\prime}}\right\rangle$ (which are very similar to each other in magnitude and distribution, as discussed in section III D); (2) a geometry-dependent term, $d \phi / d y$, which is the same for scalar and Reynolds stress, and (3) the double-averaged streamwise velocity or scalar. The scalar roughness function is smaller 

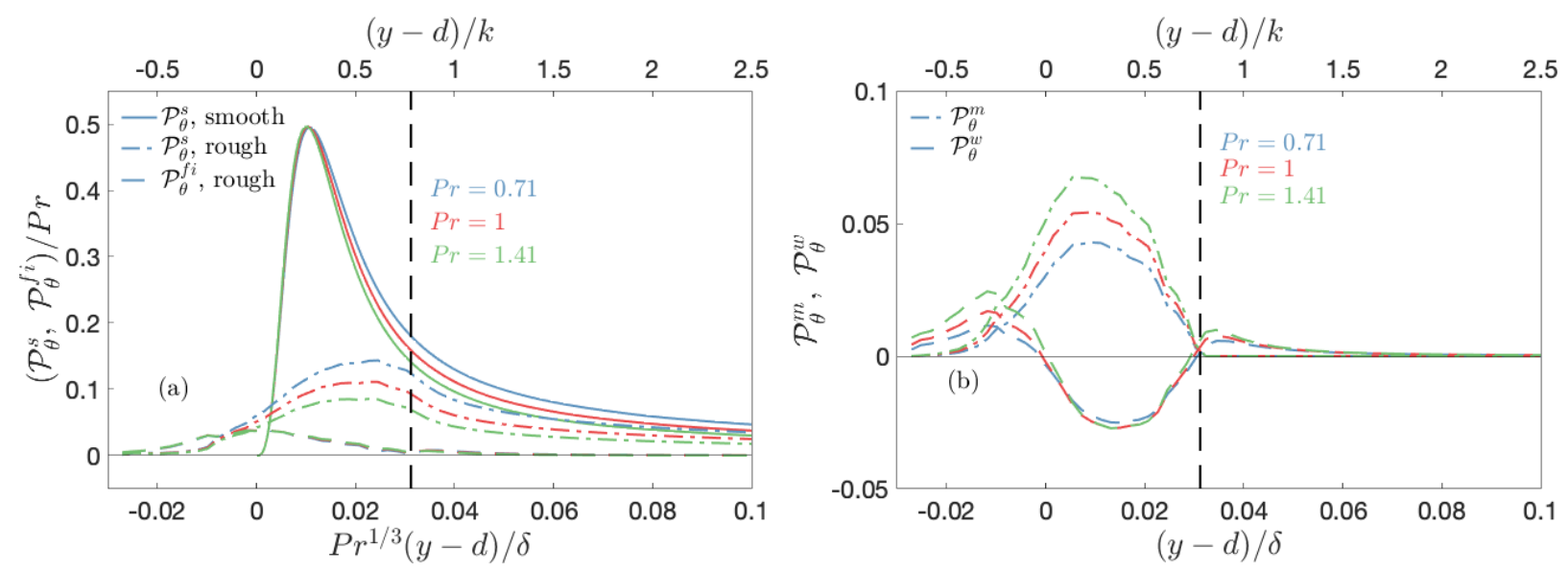

FIG. 10: Breakdown of the total production term $\mathcal{P}_{\theta}$ for different $\operatorname{Pr}$ and different wall-normal scaling. Terms are normalized by $u_{\tau}^{2} \theta_{\tau}^{2} / \nu$. The vertical line marks the roughness crest.

than the velocity one, such that $\langle\bar{\theta}\rangle^{+}>\langle\bar{u}\rangle^{+}$which results in $\mathcal{P}_{\theta}^{m}>\mathcal{P}_{11}^{m}$. Similarly, since $\langle\bar{\theta}\rangle^{+}$increases with $\operatorname{Pr}, \mathcal{P}_{\theta}^{m}$ also increases with $\operatorname{Pr}$ and, accordingly, so does $\mathcal{P}_{\theta}^{f i}$.

When considering the effect of Prandtl number on the production terms, several interesting features can be observed. For hydraulically smooth wall it was found that the (shear) production term divided by $P r$, when plotted versus $y \operatorname{Pr}^{1 / 3}$ is nearly independent of the Prandtl number [24, 54]; the peak corresponds to the transition between diffusive/conductive and turbulent fluxes, i.e., the location where $\left\langle\overline{\theta^{\prime+} v^{\prime+}}\right\rangle \simeq 0.5$. Figure 10 (a) show this behaviour for the smooth-wall case; when the wall is rough, however, this scaling does not apply. The shear production peaks further away from the wall, compared with the smooth wall, and the $\operatorname{Pr}^{-1}$ vs $\operatorname{Pr}^{1 / 3}$ scaling no longer collapses the data. The shear production of turbulent kinetic energy (or, equivalently, of $\left\langle\overline{u^{\prime} u^{\prime}}\right\rangle_{s}$ ) peaks at $y / k_{c} \simeq 0.8$, where $k_{c}$ is the roughness crest height, consistent with the results of previous studies [8, 38, 48, 55]. This also applies to $\mathcal{P}_{\theta}^{s}$. The scalar shear production no longer adheres to the smooth-wall scaling and depends on the roughness scale. This could be a result of the buffer layer being destroyed in the presence of roughness, as reported in section IIIB and the different behaviour of $d\langle\bar{\theta}\rangle / d y$ in the roughness sublayer. It is, however, interesting to note that $\mathcal{P}_{\theta}^{f i}$ scales with $\operatorname{Pr}^{-1}$ despite the fact that both of its constituting components have a clear dependence on the Prandtl number and do not scale with $\mathrm{Pr}^{-1}$, Figure 10(b). It is not clear if this behaviour has physical meaning, or if it is due to the small range of $\operatorname{Pr}$ examined 
here.

\section{F. Spatial distributions of mean and wake fields}

We now examine the spatial distribution of the various production terms. To this end, we define a "local production" $\widehat{\mathcal{P}}$, a quantity whose superficial average is equal to the corresponding production term $\mathcal{P}$, and is, therefore, defined by:

$$
\frac{1}{A_{0}} \int_{A_{f}} \widehat{\mathcal{P}} d A=\mathcal{P} .
$$

The local production components are:

$$
\begin{array}{cc}
\widehat{\mathcal{P}}_{11}=-2 \overline{u^{\prime} u_{k}^{\prime}} \frac{\partial \bar{u}}{\partial x_{k}} ; & \widehat{\mathcal{P}}_{\theta}=-2 \overline{\theta^{\prime} u_{k}^{\prime}} \frac{\partial \bar{\theta}}{\partial x_{k}} \\
\widehat{\mathcal{P}}_{11}^{s}=-2 \overline{u^{\prime} v^{\prime}} \frac{d\langle\bar{u}\rangle}{d y} ; & \widehat{\mathcal{P}}_{\theta}^{s}=-2 \overline{\theta^{\prime} v^{\prime}} \frac{d\langle\bar{\theta}\rangle}{d y} . \\
\widehat{\mathcal{P}}_{11}^{m}=-2\left\langle\overline{u^{\prime} u_{k}^{\prime}}\right\rangle \frac{\partial \widetilde{u}}{\partial x_{k}} ; & \widehat{\mathcal{P}}_{\theta}^{m}=-2\left\langle\overline{\theta^{\prime} u_{k}^{\prime}}\right\rangle \frac{\partial \theta}{\partial x_{k}} \\
\widehat{\mathcal{P}}_{11}^{w}=-2 \widetilde{u^{\prime} u_{k}^{\prime}} \frac{\partial \widetilde{u}}{\partial x_{k}} ; & \widehat{\mathcal{P}}_{\theta}^{w}=-2 \widetilde{\theta^{\prime} u_{k}^{\prime}} \frac{\partial \widetilde{\theta}}{\partial x_{k}}
\end{array}
$$

Contours of the local production terms in the $y=d$ plane are shown in Figure 11. The shear production is largest in the regions between roughness elements, reflecting the spatial distribution of the stochastic stresses and fluxes, $\overline{u^{\prime} v^{\prime}}$ and $\overline{\theta^{\prime} v^{\prime}}$ (not shown). The scalar variance and the streamwise Reynolds stress production have similar spatial distributions. The mean and wake production of the scalar have higher magnitude than the corresponding terms for the streamwise Reynolds stress, as discussed in Section IIIE. The mean production is generally positive in the stagnation regions, where the scalar gradient is large; conversely, the wake production tends to be positive in recirculation regions. The distribution of the form-induced production resembles that of its most significant term, the mean production.

Although the $x$ - component of the mean production has zero net contribution, it nonetheless plays a role in determining the distribution of the form-induced production. Figure 12 shows the contours of the five individual terms that contribute to the form-induced production of $\left\langle\overline{\theta^{\prime} \theta^{\prime}}\right\rangle_{s}$. It is interesting to note that regions of $\widehat{\mathcal{P}}_{\theta, y}^{m}>0$ (Figure $12 \mathrm{~b}$ ) tend to surround the roughness elements in a relatively isotropic way; $\widehat{\mathcal{P}}_{\theta, x}^{m}$ (Figure 12 a), on the other hand, tends to be positive in the stagnation regions, negative behind the elements, thus making 

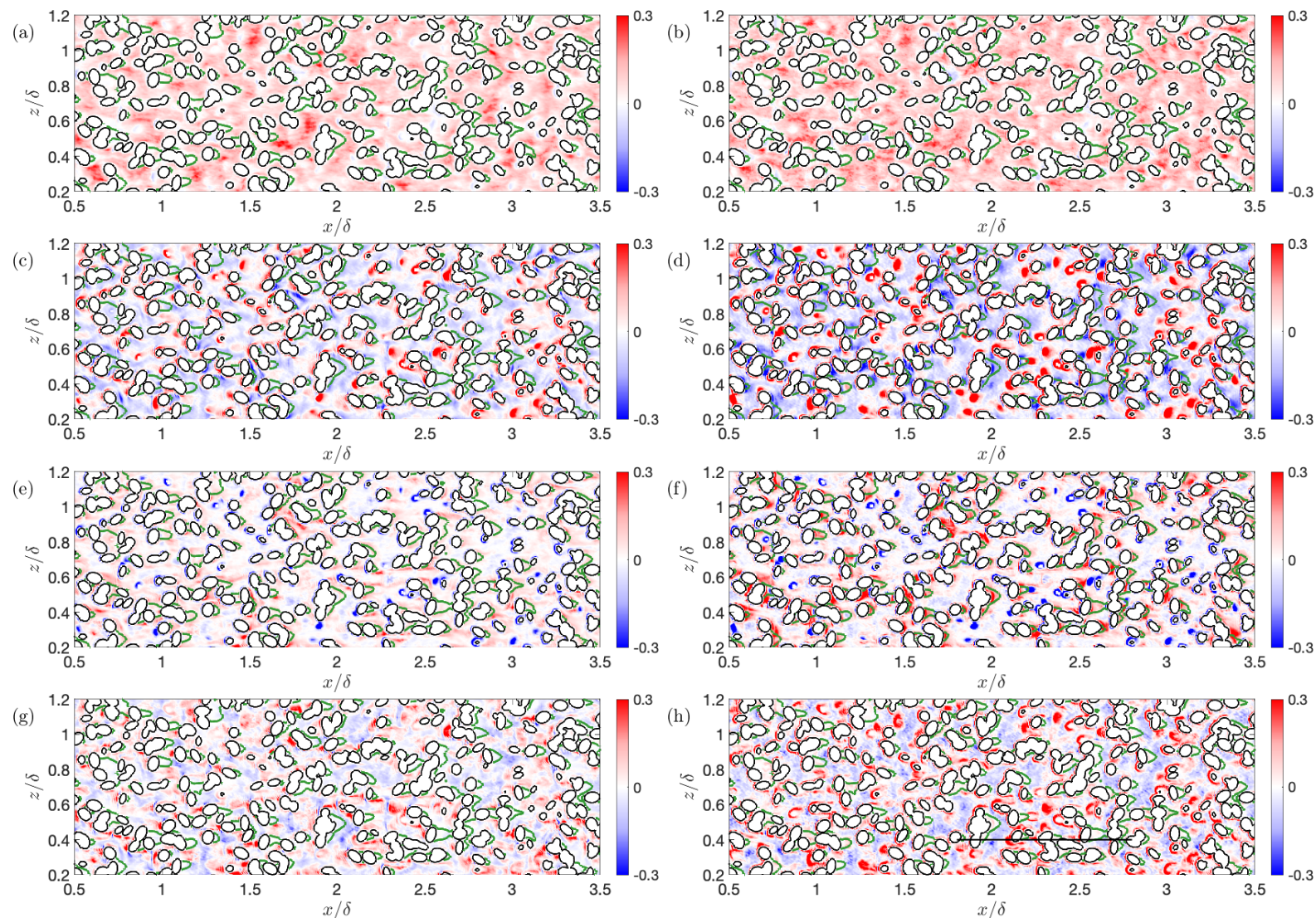

FIG. 11: Contours of production components in the $y=d$ plane. Shear production: (a)
$\widehat{\mathcal{P}}_{11}^{s} ;$
(b) $\widehat{\mathcal{P}}_{\theta}^{s}$. M
Mean production:
(c) $\widehat{\mathcal{P}}_{11}^{m}$;
(d) $\widehat{\mathcal{P}}_{\theta}^{m}$. Wake production:
(e) $\widehat{\mathcal{P}}_{11}^{w}$
(f) $\widehat{\mathcal{P}}_{\theta}^{w}$

Form-induced production: (g) $\widehat{\mathcal{P}}_{11}^{f i}$; (h) $\widehat{\mathcal{P}}_{\theta}^{f i}$. Terms are normalized by $u_{\tau}^{4} / \nu$ in the Reynolds stress budget, and $u_{\tau}^{2} \theta_{\tau}^{2} / \nu$ in the scalar one. The green line is the $\bar{u}=0$ contour. The black line in (h) indicates the location of the $x y$-plane shown in Figure 13

the $\widehat{\mathcal{P}}_{\theta}^{m}>0$ regions more prevalent near stagnation points. $\widehat{\mathcal{P}}_{\theta, y}^{m}$ appears more sensitive to the scalar transfer by diffusion, which is isotropic.

For the wake production, the local distributions of the $\widehat{\mathcal{P}}_{\theta}^{w}$ terms resemble those observed by Yuan and Aghaei Jouybari [38] for their counterpart terms of turbulent kinetic energy production. The spanwise term gives the largest positive contribution; as observed in [38] this term is associated with the convective motions in the shear layers on the sides of the roughness elements, where the fluid is deflected around the elements, thus generating spanwise velocity and scalar gradients, and consequently larger stochastic fluctuations. The $x$-component of $\widehat{\mathcal{P}}_{\theta}^{w}$, on the other hand, is positive in recirculation regions, while the $y$-component is negative in the shear layer in the front of the roughness elements. 

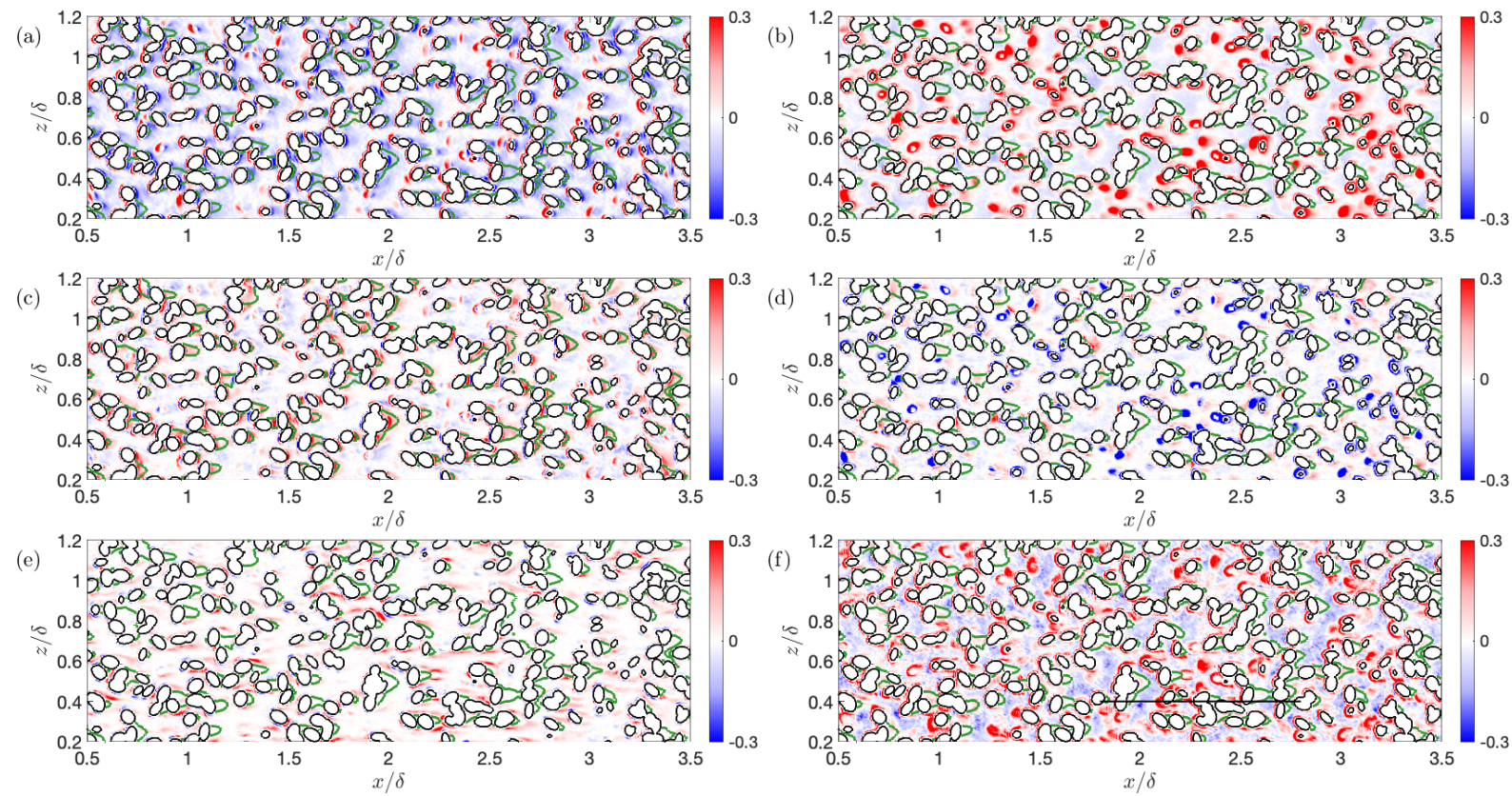

FIG. 12: Contours of $\widehat{\mathcal{P}}_{\theta}^{f i}$ and its components in the $y=d$ plane. (a) $-2\left\langle\overline{\theta^{\prime} u^{\prime}}\right\rangle(\partial \tilde{\theta} / \partial x)$; (b) $-2\left\langle\overline{\theta^{\prime} v^{\prime}}\right\rangle(\partial \widetilde{\theta} / \partial y)$; (c) $-2 \widetilde{\theta^{\prime} u^{\prime}}(\partial \tilde{\theta} / \partial x)$; (d) $-2 \widetilde{\theta^{\prime} v^{\prime}}(\partial \tilde{\theta} / \partial y)$; (e) $-2 \widetilde{\theta^{\prime} w^{\prime}}(\partial \tilde{\theta} / \partial z)$; (f) $\widehat{\mathcal{P}}_{\theta}^{f i}$. All terms are in viscous units. The green line is the $\bar{u}=0$ contour. The black line in (f) indicates the location of the $x y$-plane shown in Figure 13.

Figure 13 shows contours of several time-averaged quantities in an $x y$-plane. As observed by other researchers (e.g., [36]), a thin diffusive layer can be seen in the mean scalar distribution (highlighted by the $\bar{\theta}^{+}=1$ contour in Figure 13 (b)), but not in the mean velocity $\left(\bar{u}^{+}=1\right.$, Figure $\left.13(\mathrm{a})\right)$. The recirculation zone behind the roughness elements, however, plays a role in decreasing the convective scalar transport, and maintaining lower values of $\bar{\theta}^{+}$in those regions. Velocity and scalar gradients (and similarly, the gradients of the wake velocity and scalar, not shown) are large in the shear layers formed behind the roughness elements above the separation region, Figure $13(\mathrm{c}, \mathrm{d})$. The scalar gradient is larger than the velocity gradient in the region above lower roughness elements sheltered by taller ones. The role of sheltering in the context of momentum transfer was discussed by Millward-Hopkins et al. [56], who illustrated the effects of sheltering using examples of 'skimming flow' and 'wake interference flow'. In the context of heat transfer, Forooghi et al. [34, 35] also considered this issue. We observe here the different effect of diffusion on scalar and momentum transport: the velocity gradients are small in the regions in the shadow of the larger elements immediately upstream of them; the scalar gradient, however, is still significant in the diffusive 


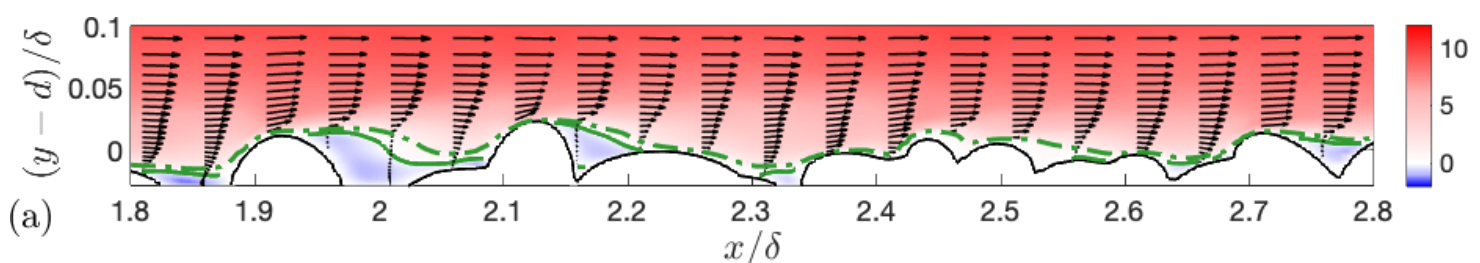

(a)

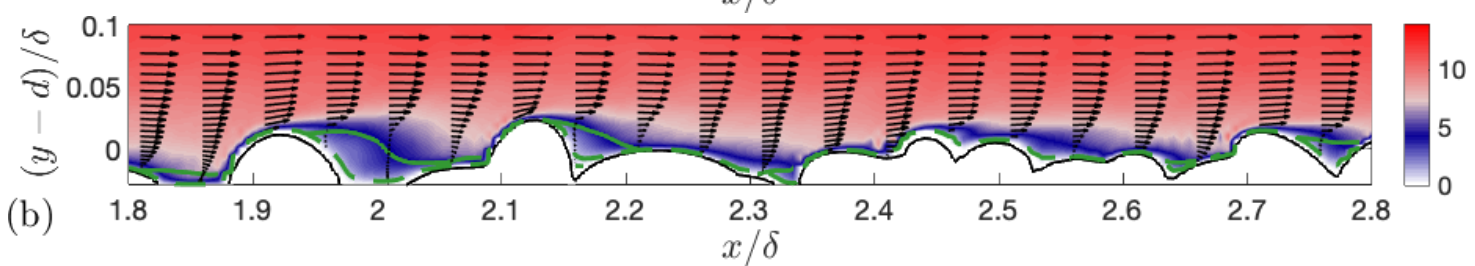

(b)

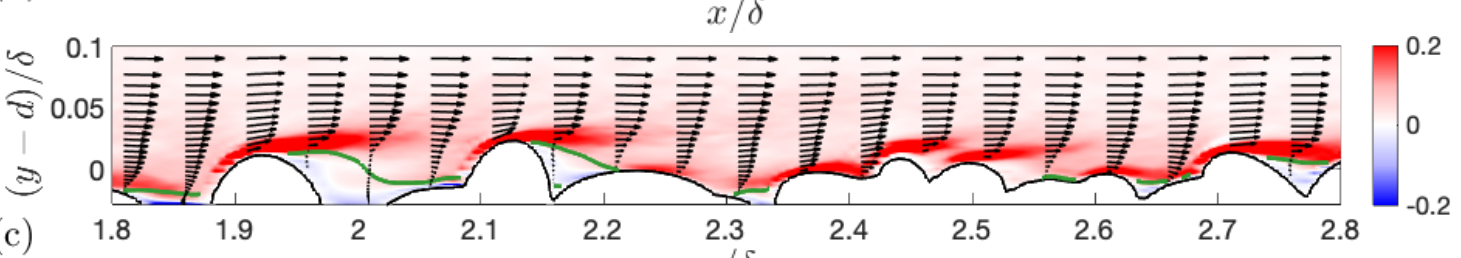

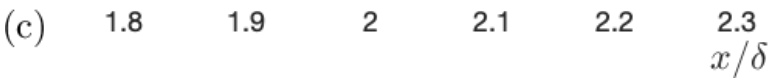
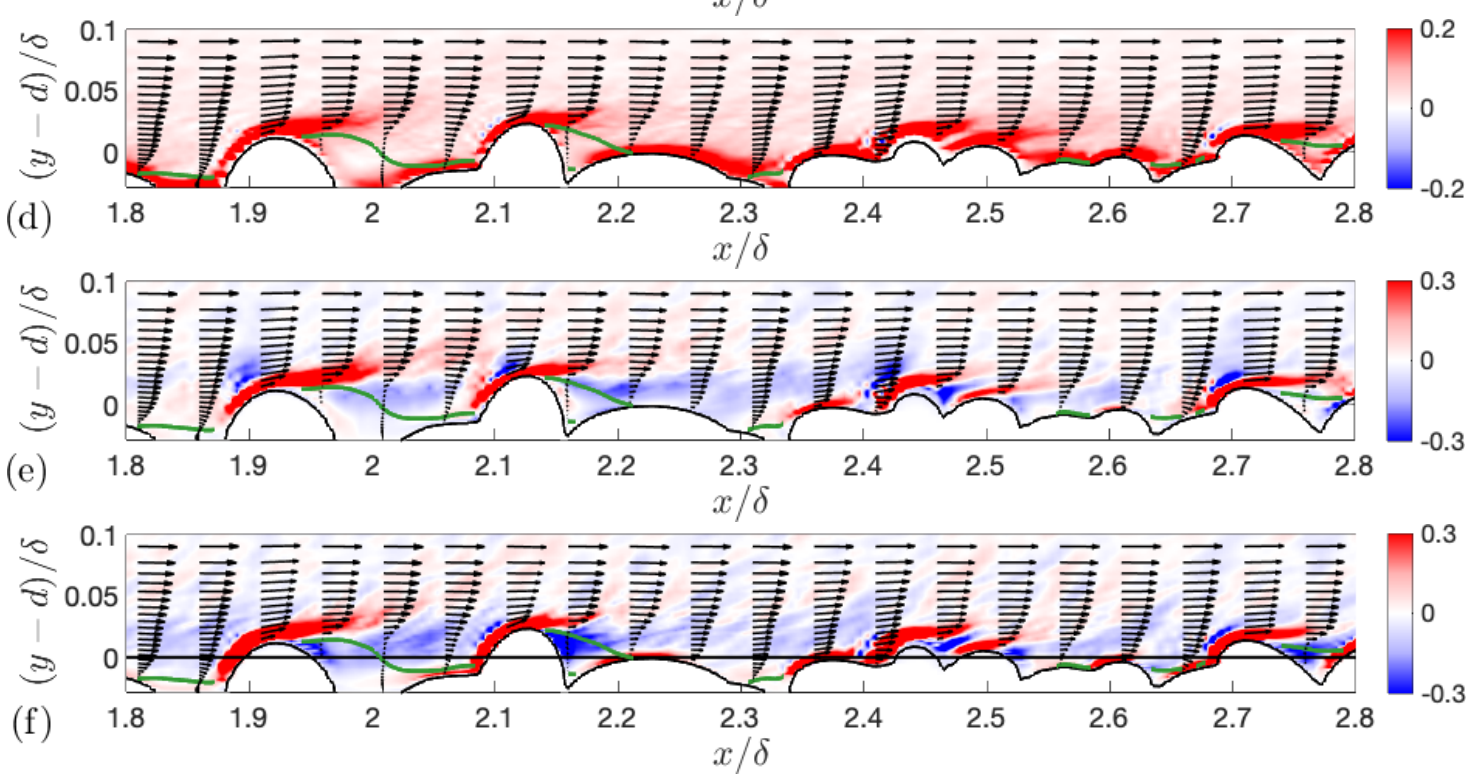

FIG. 13: Contours of (a) $\bar{u}^{+}$; (b) $\bar{\theta}^{+}$; (c) $\partial \bar{u}^{+} / \partial y$; (d) $\partial \bar{\theta}^{+} / \partial y$; (e) $\widehat{\mathcal{P}}_{11}^{m}$; (f) $\widehat{\mathcal{P}}_{\theta}^{m}$ in an $x y$-plane. The solid line is the $\bar{u}^{+}=0$ contours. The dashed lines in (a) and (b) are contours of $\bar{u}^{+}$and $\bar{\theta}^{+}=1$, respectively.

sublayer, and the regions of positive mean production are more extended for the scalar, compared with velocity. As a consequence, the regions where $\widehat{\mathcal{P}}_{\theta}^{m}>0$ are more extended than those where $\widehat{\mathcal{P}}_{11}^{m}>0$, Figure $13(\mathrm{e}, \mathrm{f})$, explaining the greater role the mean production plays in the generation of scalar fluctuations. 


\section{CONCLUSIONS}

This work studied the effects of roughness on the transport of passive scalar. Direct numerical simulations of fully-developed turbulent channel flow with smooth wall and with k-type roughness were carried out for Prandtl numbers $\operatorname{Pr}=0.71-1.41$. The simulations maintained the same mass flux and effective half-height, such that the bulk Reynolds number (based on channel half-width $\delta$ and bulk velocity $U_{b}$ ) was $R e_{b}=21,000$ for both, resulting in a Reynolds number based on friction velocity $u_{\tau}$ of $R e_{\tau}=1,020$ for the smooth-wall case and $R e_{\tau}=1,700$ for the rough-wall one.

The velocity and scalar statistics were compared and the budgets of the Reynolds stresses and scalar variance were examined, focussing on a comparison of the production terms. The triple decomposition was employed to distinguish form-induced contributions and separate the direct and indirect effects of roughness.

The scalar exhibits a downwards shift in the log-law region (the roughness function $\Delta \Theta^{+}$), similar to the velocity shift but smaller in magnitude. This result is consistent with the observations of other researchers [36, 37]. The scalar roughness function increases with $\operatorname{Pr}$.

The mean wall stress is dominated by the form-induced pressure $F_{p}$; the form-induced viscous contribution $F_{\nu}$ accounts for approximately $30 \%$ of the wall stress. The mean scalar wall-flux, conversely, is almost entirely due to the form-induced diffusive flux $F_{\alpha}$ (equivalent to $F_{\nu}$ ), as there is no mechanism analogous to the pressure drag. $F_{\alpha}$ follows the solid fraction $1-\phi$ closely, emphasizing the importance of the wetted area for the scalar flux. At the virtual wall $y=d$, the form-induced terms $F_{p}, F_{\nu}$ and the dispersive stress $\langle\widetilde{u} \widetilde{v}\rangle_{s}$ account for more than half of the momentum flux.

The dispersive flux $\langle\widetilde{\theta} \widetilde{v}\rangle_{s}^{+}$is similar, qualitatively and quantitatively, to the dispersive stress $\langle\widetilde{u} \widetilde{v}\rangle_{s}^{+}$. For $\operatorname{Pr}=1.0$ the dispersive flux is slightly higher than the dispersive stress. Dispersive fluxes and stresses are significant in the roughness sublayer, and are of the same order as (or larger than) the stochastic stresses and fluxes, in line with the findings of [31].

The roughness significantly dampens the intensity of streamwise fluctuations $\left\langle\overline{u^{\prime} u^{\prime}}\right\rangle_{s}^{+}$, compared with the smooth-wall case. The scalar fluctuation variance, $\left\langle\overline{\theta^{\prime} \theta^{\prime}}\right\rangle_{s}^{+}$, is reduced by a smaller amount, which also depends on the Prandtl number; the streamwise turbulent flux $\left\langle\overline{\theta^{\prime} u^{\prime}}\right\rangle_{s}^{+}$mirrored the behaviour of $\left\langle\overline{u^{\prime} u^{\prime}}\right\rangle_{s}^{+}$. The rough-wall scalar statistics collapse on their smooth-wall equivalent several roughness-heights from the wall, suggesting that Townsend's 
similarity hypothesis also applies to scalar when $\operatorname{Pr}$ is near unity.

For the rough-wall case, the pressure-work $\Pi_{11}$ and dissipation $\varepsilon_{11}$ are equally important sink terms in the budget of $\left\langle\overline{u^{\prime} u^{\prime}}\right\rangle_{s}$. For $\left\langle\overline{\theta^{\prime} \theta^{\prime}}\right\rangle_{s}$, however, $\varepsilon_{\theta}$ is the only significant sink term, roughly equal to the sum of magnitudes of $\Pi_{11}$ and $\varepsilon_{11}$. The shear production of $\left\langle\overline{\theta^{\prime} \theta^{\prime}}\right\rangle_{s}$ and $\left\langle\overline{u^{\prime} u^{\prime}}\right\rangle_{s}$ are similar in smooth- and rough-wall cases. The presence of roughness dampens both of the shear production terms in a similar manner; however the shear production remains the leading source term away from the wall.

The form-induced production plays a very significant role in the budget of the scalar fluctuation variance, $\left\langle\overline{\theta^{\prime} \theta^{\prime}}\right\rangle_{s}^{+}$, more so than in the budget of $\left\langle\overline{u^{\prime} u^{\prime}}\right\rangle_{s}^{+}$. Up to the location of the virtual wall, $y=d, \mathcal{P}_{\theta}^{f i}$ gives the largest positive contribution to the budget. The form-induced production has two components: mean and wake production. Generally, the two are opposite in sign, highly anti-correlated and the mean production $\mathcal{P}^{m}$ is greater in magnitude. $\mathcal{P}^{m}$ depends explicitly on the geometry and is identically zero above the roughness crest, while the wake production $\mathcal{P}^{w}$ is related to the geometry indirectly, through the adjustment of the mean flow-field. The mean production terms for momentum and scalar are proportional to the mean velocity $\langle\bar{u}\rangle^{+}$and mean scalar $\langle\bar{\theta}\rangle^{+}$, respectively. Since, for the range of $\operatorname{Pr}$ examined, $\langle\bar{u}\rangle^{+}\left\langle\langle\bar{\theta}\rangle^{+}\right.$, the mean production of $\left\langle\overline{\theta^{\prime} \theta^{\prime}}\right\rangle_{s}$ is larger than that of $\left\langle\overline{u^{\prime} u^{\prime}}\right\rangle_{s}$. Accordingly, since $\langle\bar{\theta}\rangle^{+}$increases with $\operatorname{Pr}$, the mean production $\mathcal{P}_{\theta}^{m}$ also increases with $\operatorname{Pr}$ as does the form-induced production $\mathcal{P}_{\theta}^{f i}$.

An examination of the (spatially) local contributions to the form-induced production highlights several interesting features. First, as expected and noted by other researchers, the scalar distribution (but not the velocity) shows the presence of a diffusive sublayer; however, the separation behind the taller roughness elements affects the convective scalar transfer, so that in recirculation regions the scalar gradients are reduced. The spatial distribution of form-induced production is generally similar for scalar and velocity fluctuations. Diffusion, however, plays an important role in the form-induced production: while the velocity gradient is negligible behind the larger roughness elements, the scalar gradient remains significant because of the thin diffusive sublayer formed around the roughness elements themselves. This effect increases the form-induced production, resulting in larger scalar variance, compared to the streamwise Reynolds stress.

The breakdown of the Reynolds Analogy has long been attributed to the difference between the more advective character of momentum, compared with scalar transport, in which 
diffusion plays a larger role. The form-induced production is a manifestation of this role. In the scalar transport, the diffusive sublayer results in significant scalar gradients on lower roughness elements, which are in the shadow of larger ones. The velocity gradients, on the other hand, remain small because of the recirculation behind the tall elements. These larger gradients result in larger form-induced production and, consequently, in larger scalar variance.

Our observations on the different effects the roughness geometry has on the scalar and velocity fluctuations highlight the role of the geometry in the generation of fluctuations of the scalar, and may have some significance for developers of turbulence models. The only parameter that characterizes the roughness geometry in turbulence models is the equivalent sandgrain roughness, $k_{s}^{+}$, which is related to the roughness function. While this relation, for the momentum transport, is well established, and several experimental correlations are available, for the scalar roughness function the situation is quite different. The results of [36, 37], as well as our own, show significant scatter of the data and Prandtl-number dependency. The lack of a universal expression is particularly important because our work indicates that the mechanisms governing the production of scalar fluctuations are significantly different than those governing the production of TKE, and that the effect of the geometry (which causes the form-induced production) is much more significant. Thus, the definition of a "scalar equivalent sandgrain roughness" appears desirable. Additional geometrical parameters may also be needed to model the effects of the roughness shape.

The present work examined a single roughness height and type, considered a moderate Reynolds number and Prandtl numbers of order one. It would be interesting to determine how the Prandtl number affects the scalar transport mechanisms. The effective slope of the surface could also affect the scalar transport, as the sheltering would probably be less significant for surfaces in the wavy regime than for those in the rough regime. Thus, different topographical features of the roughness could play a role. In addition, the specific behaviours of the form-induced production terms could suggest improved models. These topics will be the subject of future studies. 


\section{ACKNOWLEDGEMENTS}

UP acknowledges the support from the Natural Science and Engineering Research Council of Canada (NSERC) under the Discovery Grant program. UP also acknowledges the support of the Canada Research Chair program. ZH acknowledges the support of the Ontario Trillium Scholarship (OTS) provided by the Government of Ontario. This research was enabled in part by computational support provided by Compute Ontario (computeontario.ca) and Compute Canada (www.computecanada.ca). 
[1] O. Reynolds, On the extent and action of the heating surface for steam boilers, Manchester Lit. Phil. Soc. (1874).

[2] W. M. Kays and M. E. Crawford, Convective heat and mass transfer, Vol. 76 (McGraw-Hill Higher Education Boston, 2005).

[3] A. P. Colburn, Relation between mass transfer (absorption) and fluid friction, Ind. \& Eng. Chem. 22, 967 (1930).

[4] T. H. Chilton and A. P. Colburn, Mass transfer (absorption) coefficients prediction from data on heat transfer and fluid friction, Ind. \& Eng. Chem. 26, 1183 (1934).

[5] W. F. Cope, The friction and heat transmission coefficients of rough pipes, Proc. Inst. Mech. Eng. 145, 99 (1941).

[6] W. Nunner, Heat transfer and pressure drop in rough tubes (Atomic Energy Research Establishment, 1958).

[7] R. T. Lancet, The effect of surface roughness on the convection heat-transfer coefficient for fully developed turbulent flow in ducts with uniform heat flux, J. Heat Tran. 81, 168 (1959).

[8] M. R. Raupach, R. A. Antonia, and S. Rajagopalan, Rough-wall boundary layers, App. Mech Rev. 44, 1 (1991).

[9] J. Jiménez, Turbulent flows over rough walls, Annu. Rev. Fluid Mech. 36, 173 (2004).

[10] J. Nikuradse, Laws of flow in rough pipes, english translation 1950, Papers on Mechanical and Physical Subjects 1 (1933).

[11] C. F. Colebrook, Turbulent flow in pipes with particular reference to the transition region between smooth- and rough-pipe laws, J. Inst. Civ. Eng. 11, 133 (1939).

[12] P.-Å. Krogstad, R. A. Antonia, and L. W. B. Browne, Comparison between rough-and smoothwall turbulent boundary layers, J. Fluid Mech. 245, 599 (1992).

[13] H. S. Shafi and R. A. Antonia, Anisotropy of the reynolds stresses in a turbulent boundary layer on a rough wall, Exp. Fluids 18, 213 (1995).

[14] P.-Å. Krogstad and R. A. Antonia, Surface roughness effects in turbulent boundary layers, Exp. Fluids 27, 450 (1999).

[15] J. Finnigan, Turbulence in plant canopies, Annu. Rev. Fluid Mech. 32, 519 (2000).

[16] A. A. Townsend, The structure of turbulent shear flow (Cambridge university press, 1980). 
[17] K. A. Flack, M. P. Schultz, and T. A. Shapiro, Experimental support for townsend's reynolds number similarity hypothesis on rough walls, Phys. Fluids 17, 035102 (2005).

[18] P. R. Owen and W. R. Thomson, Heat transfer across rough surfaces, J. Fluid Mech. 15, 321 (1963).

[19] D. F. Dipprey and R. H. Sabersky, Heat and momentum transfer in smooth and rough tubes at various prandtl numbers, Int. J. Heat and Mass Trans. 6, 329 (1963).

[20] A. Yaglom and B. Kader, Heat and mass transfer between a rough wall and turbulent fluid flow at high reynolds and peclet numbers, J. Fluid Mech. 62, 601 (1974).

[21] H. Schlichting, Experimentelle untersuchungen zum rauhigkeitsproblem, Arch. App. Mech. 7, 1 (1936).

[22] J. Kim and P. Moin, Transport of passive scalars in a turbulent channel flow, in Turbulent Shear Flows 6 (Springer, 1989) pp. 85-96.

[23] N. Kasagi, Y. Tomita, and A. Kuroda, Direct numerical simulation of passive scalar field in a turbulent channel flow, J. Heat Tran. 114, 598 (1992).

[24] H. Kawamura, K. Ohsaka, H. Abe, and K. Yamamoto, Dns of turbulent heat transfer in channel flow with low to medium-high prandtl number fluid, Int. J. Heat and Fluid Flow 19, $482(1998)$.

[25] H. Kawamura, H. Abe, and K. Shingai, Dns of turbulence and heat transport in a channel flow with different reynolds and prandtl numbers and boundary conditions, J. Turb. Heat and Mass Tran. 3, 15 (2000).

[26] H. Abe, H. Kawamura, and Y. Matsuo, Surface heat-flux fluctuations in a turbulent channel flow up to $\operatorname{re} \tau=1020$ with pr=0.025 and 0.71, Int. J. Heat and Fluid Flow 25, 404 (2004).

[27] H. Abe, R. Antonia, and H. Kawamura, Correlation between small-scale velocity and scalar fluctuations in a turbulent channel flow, J. Fluid Mech. 627, 1 (2009).

[28] R. A. Antonia, H. Abe, and H. Kawamura, Analogy between velocity and scalar fields in a turbulent channel flow, J. Fluid Mech. 628, 241 (2009).

[29] S. Pirozzoli, M. Bernardini, and P. Orlandi, Passive scalars in turbulent channel flow at high reynolds number, J. Fluid Mech. 788, 614 (2016).

[30] Y. Nagano, H. Hattori, and T. Houra, DNS of velocity and thermal fields in turbulent channel flow with transverse-rib roughness, Int. J. Heat Fluid Flow 25, 393 (2004) 
[31] S. Leonardi, P. Orlandi, L. Djenidi, and R. A. Antonia, Heat transfer in a turbulent channel flow with square bars or circular rods on one wall, J. Fluid Mech. 776, 512 (2015).

[32] P. Orlandi, D. Sassun, and S. Leonardi, DNS of conjugate heat transfer in presence of rough surfaces, Int. J. Heat Mass Trans. 100, 250 (2016).

[33] Q. Li and E. Bou-Zeid, Contrasts between momentum and scalar transport over very rough surfaces, J. Fluid Mech. 880, 32 (2019).

[34] P. Forooghi, A. Weidenlener, F. Magagnato, B. Böhm, H. Kubach, T. Koch, and B. Frohnapfel, DNS of momentum and heat transfer over rough surfaces based on realistic combustion chamber deposit geometries, Int. J. Heat Fluid Flow 69, 83 (2018).

[35] P. Forooghi, M. Stripf, and B. Frohnapfel, A systematic study of turbulent heat transfer over rough walls, Int. J. Heat Mass Trans. 127, 1157 (2018).

[36] M. MacDonald, N. Hutchins, and D. Chung, Roughness effects in turbulent forced convection, Journal of Fluid Mechanics 861, 138 (2019).

[37] J. W. R. Peeters and N. D. Sandham, Turbulent heat transfer in channels with irregular roughness, Int. J. Heat Mass Trans. 138, 454 (2019).

[38] J. Yuan and M. Aghaei Jouybari, Topographical effects of roughness on turbulence statistics in roughness sublayer, Phys. Rev. Fluids 3, 114603 (2018).

[39] N. Kasagi, A. Kuroda, and M. Hirata, Numerical investigation of near-wall turbulent heat transfer taking into account the unsteady heat conduction in the solid wall, J. Heat Tran. 111, 385 (1989).

[40] A. Scotti, Direct numerical simulation of turbulent channel flows with boundary roughened with virtual sandpaper, Phys. Fluids 18, 031701 (2006).

[41] A. J. Chorin, Numerical solution of the navier-stokes equations, Math. Comput. 22, 745 (1968).

[42] J. Kim and P. Moin, Application of a fractional-step method to incompressible navier-stokes equations, J. Comput. Phys. 59, 308 (1985).

[43] J. Yuan and U. Piomelli, Estimation and prediction of the roughness function on realistic surfaces, J. Turbulence 15, 350 (2014).

[44] J. Yuan and U. Piomelli, Roughness effects on the Reynolds stress budgets in near-wall turbulence, J. Fluid Mech. 760, R1 (2014),

[45] J. Yuan and U. Piomelli, Numerical simulations of sink-flow boundary layers over rough surfaces, Phys. Fluids 26, 015113 (2014). 
[46] V. Nikora, S. McLean, S. Coleman, D. Pokrajac, I. McEwan, L. Campbell, J. Aberle, D. Clunie, and K. Koll, Double-averaging concept for rough-bed open-channel and overland flows: Applications, J. Hydr. Eng. 133, 884 (2007).

[47] M. R. Raupach and R. H. Shaw, Averaging procedures for flow within vegetation canopies, Bound.-Lay. Meteorol. 22, 79 (1982).

[48] E. Mignot, E. Barthelemy, and D. Hurter, Double-averaging analysis and local flow characterization of near-bed turbulence in gravel-bed channel flows, J. Fluid Mech. 618, 279 (2009).

[49] P. S. Jackson, On the displacement height in the logarithmic velocity profile, J. Fluid Mech. 111, 15 (1981).

[50] R. A. Antonia and P.-Å. Krogstad, Turbulence structure in boundary layers over different types of surface roughness, Fluid Dyn. Res. 28, 139 (2001).

[51] R. J. Smalley, S. Leonardi, R. A. Antonia, L. Djenidi, and P. Orlandi, Reynolds stress anisotropy of turbulent rough wall layers, Exp. Fluids 33, 31 (2002).

[52] K. A. Flack and M. P. Schultz, Roughness effects on wall-bounded turbulent flows, Phys. Fluids 26, 101305 (2014).

[53] O. Coceal, T. Thomas, I. Castro, and S. Belcher, Mean flow and turbulence statistics over groups of urban-like cubical obstacles, Bound.-Lay. Meteorol. 121, 491 (2006).

[54] M. Teitel and R. Antonia, Heat transfer in fully developed turbulent channel flow: comparison between experiment and direct numerical simulations, Int. J. Heat Mass Trans. 36, 1701 (1993).

[55] F. López and M. H. García, Mean flow and turbulence structure of open-channel flow through non-emergent vegetation, J. Hydr. Eng. 127, 392 (2001).

[56] J. Millward-Hopkins, A. Tomlin, L. Ma, D. Ingham, and M. Pourkashanian, Estimating aerodynamic parameters of urban-like surfaces with heterogeneous building heights, Boundarylayer meteorology 141, 443 (2011). 


\section{Appendix A: Grid refinement}

A grid-refinement study was conducted to determine whether the mesh used is sufficient to resolve the momentum and scalar transport, particularly for the case of $\operatorname{Pr}=1.4$, in which a wider range of scales is present for the scalar than for momentum. This study was conducted for the smooth-wall case, in which diffusive effects are more significant. For rough, wall-bounded flows the eddy size is determined by roughness geometry rather than by diffusive phenomena. Thus, restrictions on near-wall resolution (smallest scales) are determined by the roughness scale and, for high Re case, are less strict than for smooth-wall cases. A resolution of $\Delta x=\Delta z \simeq k / 7$ is sufficient to resolve the momentum [45] in the roughness region and, since the geometry is independent of $P r$, should also suffice for the scalars. Note that the elements themselves (i.e., the ellipsoid semi-axes) are larger than the roughness length scale $k$, so that one roughness element is actually resolved by more than 7 points in each direction.

We performed calculations on a coarser mesh using $720 \times 320 \times 360$ cells, 1.4 times coarser, in the homogeneous directions, than the mesh used throughout the study (which had $1024 \times 320 \times 512$ cells). Figure 14 shows a comparison of the statistics obtained. The mean scalar and velocity compare very well: the difference between fine- and coarse-mesh results (normalized by the fine-grid value) is less than 3\%. Making the reasonable assumption that the error has the same sign for smooth and rough walls (for instance, the log-law intercept is overestimated in both cases) would result in an uncertainty of $2 \%$ for $\Delta U^{+}$and $3.2 \%$, $4.2 \%$ and $4.5 \%$ for $\Delta \Theta^{+}$with $\operatorname{Pr}=0.71,1.0$ and 1.41 respectively. In Figure 3, the symbols used are larger than the error bars would be. If the errors were in opposite directions the uncertainty would be twice as large but still acceptable, and only slightly larger than the symbols.

The scalar variance $\left\langle\overline{\theta^{\prime} \theta^{\prime}}\right\rangle_{s}$ and streamwise turbulent flux $\left\langle\overline{\theta^{\prime} u^{\prime}}\right\rangle_{s}$ are also in good agreement, the main difference being a small shift of their peaks, probably due to the larger size of the eddies in the coarse-mesh calculation. 

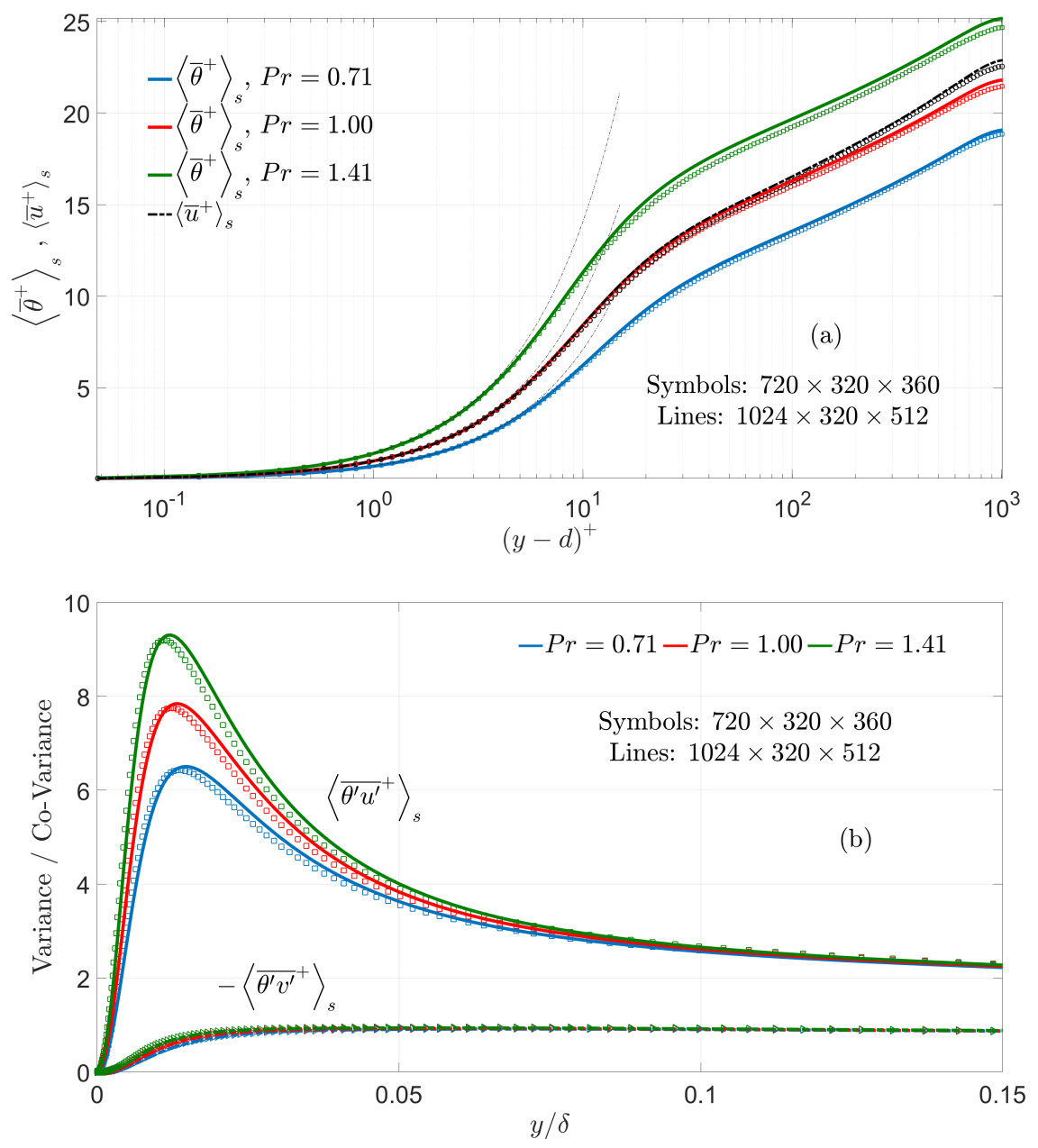

FIG. 14: Grid refinement study: (a) Mean scalar $\langle\bar{\theta}\rangle_{s}$ and streamwise velocity $\langle\bar{u}\rangle_{s}$; (b) Scalar variance $\left\langle\overline{\theta^{\prime} \theta^{\prime}}\right\rangle_{s}$ and streamwise flux $\left\langle\overline{\theta^{\prime} u_{i}^{\prime}}\right\rangle_{s}$. Markers: coarse mesh; lines: fine mesh. Colors: Blue $\operatorname{Pr}=0.71$; Red $\operatorname{Pr}=1.0$; Green $\operatorname{Pr}=1.41$.

\section{Appendix B: Reynolds stress and flux budgets}

The budget of the double-averaged Reynolds stresses can be written as

$$
0=\mathcal{T}_{i j}+\mathcal{A}_{i j}+\mathcal{D}_{i j}+\mathcal{P}_{i j}+\Pi_{i j}-\varepsilon_{i j}
$$

where the terms on the RHS are the turbulent transport, wake transport, viscous diffusion, production, pressure work (or velocity-pressure-gradient) and the dissipation, respectively. 
They are defined by:

$$
\begin{aligned}
\mathcal{T}_{i j} & =-\left\langle\frac{\partial}{\partial x_{k}}\left(\overline{u_{i}^{\prime} u_{j}^{\prime} u_{k}^{\prime}}\right)\right\rangle_{s} \\
\mathcal{A}_{i j} & =-\left\langle\frac{\partial}{\partial x_{k}}\left(\widetilde{u_{i}^{\prime} u_{j}^{\prime}} \widetilde{u}_{k}\right)\right\rangle_{s} \\
\mathcal{D}_{i j} & =\nu\left\langle\nabla^{2}\left(\overline{u_{i}^{\prime} u_{j}^{\prime}}\right)\right\rangle_{s} \\
\mathcal{P}_{i j} & =-\left(\left\langle\overline{u_{i}^{\prime} u_{k}^{\prime}} \frac{\partial \overline{u_{j}}}{\partial x_{k}}\right\rangle_{s}+\left\langle\overline{u_{j}^{\prime} u_{k}^{\prime}} \frac{\partial \overline{u_{i}}}{\partial x_{k}}\right\rangle_{s}\right) \\
\Pi_{i j} & \left.=-\frac{1}{\rho}\left[\overline{\left\langle\overline{u_{i}^{\prime}} \frac{\partial p^{\prime}}{\partial x_{j}}\right.}\right\rangle_{s}+\left\langle\overline{u_{j}^{\prime} \frac{\partial p^{\prime}}{\partial x_{i}}}\right\rangle_{s}\right]_{s} \\
\varepsilon_{i j} & =2 \nu\left\langle\frac{\partial u_{i}^{\prime}}{\partial x_{k}} \frac{\partial u_{j}^{\prime}}{\partial x_{k}}\right\rangle_{s}
\end{aligned}
$$

In a similar fashion, the budget of the scalar variance can be written as:

$$
0=\mathcal{T}_{\theta}+\mathcal{A}_{\theta}+\mathcal{D}_{\theta}+\mathcal{P}_{\theta}+Q_{\theta}-\varepsilon_{\theta}
$$

where the terms on the RHS are the turbulent transport, wake transport, viscous diffusion, production, scalar source contribution and the dissipation, respectively and are defined by:

$$
\begin{aligned}
\mathcal{T}_{\theta} & =-\left\langle\frac{\partial}{\partial x_{k}}\left(\overline{\theta^{\prime} \theta^{\prime} u_{k}^{\prime}}\right)\right\rangle_{s} \\
\mathcal{A}_{\theta} & =-\left\langle\frac{\partial}{\partial x_{k}}\left(\widetilde{\theta^{\prime} \theta^{\prime}} \widetilde{u}_{k}\right)\right\rangle_{s} \\
\mathcal{D}_{\theta} & =\alpha\left\langle\nabla^{2}\left(\overline{\theta^{\prime} \theta^{\prime}}\right)\right\rangle_{s} \\
Q_{\theta} & =\left\langle\overline{\theta^{\prime} Q^{\prime}}\right\rangle_{s}=\frac{q_{w}}{\rho c_{p} \delta \mathrm{U}_{b}}\left\langle\overline{\theta^{\prime} u^{\prime}}\right\rangle_{s} \\
\varepsilon_{\theta} & =2 \alpha\left\langle\overline{\left.\frac{\partial \theta^{\prime}}{\partial x_{i}} \frac{\partial \theta^{\prime}}{\partial x_{j}}\right\rangle_{s}}\right.
\end{aligned}
$$

The source term contribution $Q_{\theta}$ is the interaction of the fluctuating part of the scalar source term $Q^{\prime}$ with the fluctuating scalar $\theta^{\prime}$. The terms in Eq. (B2) and Eq. (B4) can be further simplified using commutation rules presented in [46].

Figure 8 in section III E presented only the significant terms in the $\left\langle\overline{u^{\prime} u^{\prime}}\right\rangle_{s}$ and $\left\langle\overline{\theta^{\prime} \theta^{\prime}}\right\rangle_{s}$ budgets. Figure 15 includes all the budget terms over a smaller portion of the domain for improved visibility. 

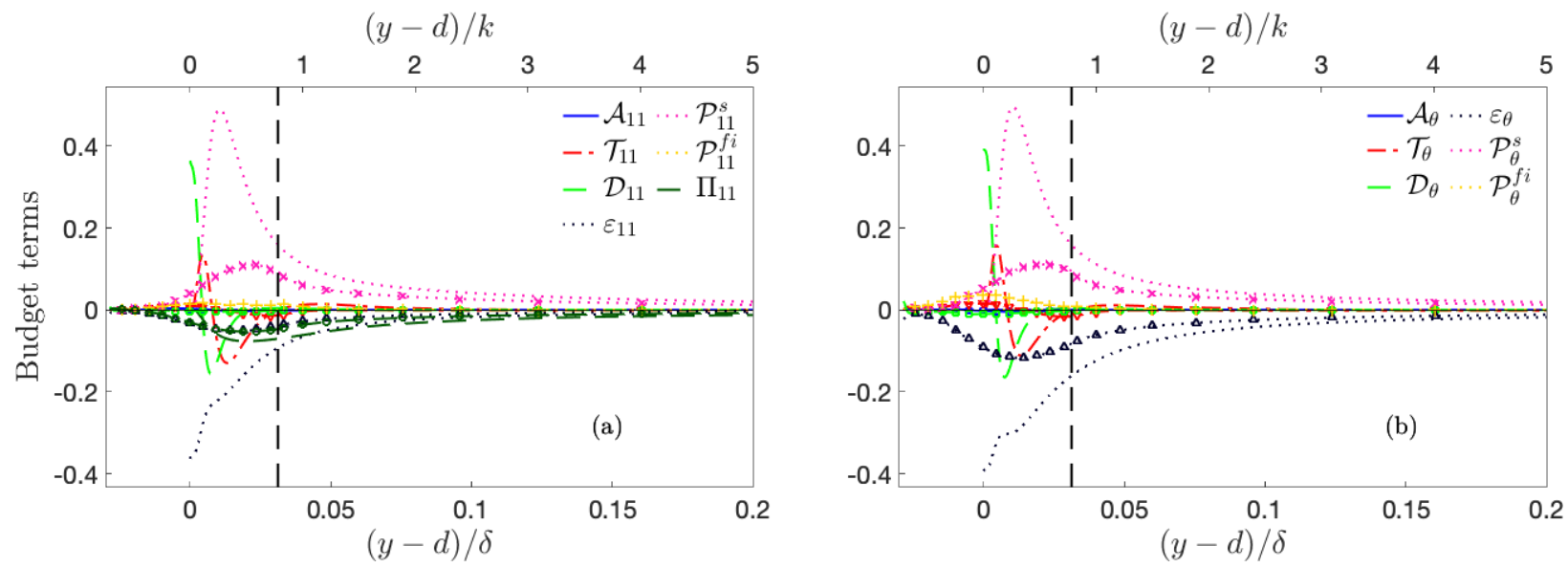

FIG. 15: Budgets of $(\mathrm{a})\left\langle\overline{u^{\prime} u^{\prime}}\right\rangle_{s}$ and $(\mathrm{b})\left\langle\overline{\theta^{\prime} \theta^{\prime}}\right\rangle_{s}$ for $\operatorname{Pr}=1.0$. Lines: Smooth wall; Markers: rough wall. Budget terms in (a) and (b) are normalized by $u_{\tau}^{4} / \nu$, and $u_{\tau}^{2} \theta_{\tau}^{2} / \nu$, respectively. The vertical line marks the roughness crest. 